\title{
On Calculating Deposition Coefficients and Aspect-Ratio Evolution in Approximate Models of Ice Crystal Vapor Growth
}

\author{
Jerry Y. Harrington, Alfred Moyle, and Lavender Elle Hanson \\ Department of Meteorology and Atmospheric Science, The Pennsylvania State University, University Park, Pennsylvania \\ HUGH MORRISON \\ National Center for Atmospheric Research, Boulder, Colorado
}

(Manuscript received 23 October 2018, in final form 8 February 2019)

\begin{abstract}
Models of ice crystal vapor growth require estimates of the deposition coefficient $\alpha$ when surface attachment kinetics limit growth and when ice crystal shape is predicted. Parametric models can be used to calculate $\alpha$ for faceted growth as long as characteristic supersaturation $s_{\text {char }}$ values are known. However, previously published measurements of $s_{\text {char }}$ are limited to temperatures higher than $-40^{\circ} \mathrm{C}$. Estimates of $s_{\text {char }}$ at temperatures between $-40^{\circ}$ and $-70^{\circ} \mathrm{C}$ are provided here through reanalysis of vapor growth data. The estimated $s_{\text {char }}$ follow the same functional temperature dependence as data taken at higher temperatures. Polynomial fits to $s_{\text {char }}$ are used as inputs to a parameterization of $\alpha$ suitable for use in cloud models. Comparisons of the parameterization with wind tunnel data show that growth at liquid saturation and constant temperatures between $-3^{\circ}$ and $-20^{\circ} \mathrm{C}$ can be modeled by ledge nucleation for larger (hundreds of micrometers) crystals; however, comparisons with free-fall chamber data at $-7^{\circ} \mathrm{C}$ suggest that dislocation growth may be required to model the vapor growth of small crystals $(\sim 20 \mu \mathrm{m})$ at liquid saturation. The comparisons with free-fall chamber data also show that the parameterization can reproduce the measured pressure dependence of aspect-ratio evolution. Comparisons with a hexagonal growth model indicate that aspect-ratio evolution based on the theory of Chen and Lamb produces unrealistically fast column growth near $-7^{\circ} \mathrm{C}$ that is mitigated if a theory based on faceted growth is used. This result indicates that the growth hypothesis used in habit-evolving microphysical models needs to be revised when deposition coefficients are predicted.
\end{abstract}

\section{Introduction}

Cold cloud systems are sensitive to the manner in which ice vapor growth is parameterized (Gierens et al. 2003; Avramov and Harrington 2010), and while our knowledge is sufficient to formulate approximate models the mechanisms controlling ice crystal growth remain poorly understood. Laboratory data for vapor grown ice crystals exist at temperatures above $-40^{\circ} \mathrm{C}$, but the quantities measured in many laboratory studies (Nelson and Knight 1998; Libbrecht 2003b) are often not amenable to direct inclusion in the capacitance analogy that is almost universally used in atmospheric applications. This has led to an unfortunate situation in which the methods used to represent ice growth in atmospheric models are almost entirely divorced from

\footnotetext{
Corresponding author: Jerry Y. Harrington, jyh10@psu.edu
}

process-oriented measurements. While popular parameterization methods have difficulties reproducing laboratory measurements (Westbrook and Heymsfield 2011; Harrington et al. 2013b), a more fundamental issue is that these methods do not account for the growth of faceted ice. Popular parameterizations are rooted in capacitance theory, which assumes that the vapor density is constant over the crystal surface. The aspect ratio cannot evolve in this model (Nelson 1994, 83-85) unless it is supplemented with an auxiliary hypothesis (Chen and Lamb 1994). In contrast, faceting requires a uniform flux boundary condition. Moreover, faceting indicates that crystal evolution is controlled by surface attachment kinetics (attachment kinetics) that are supersaturation dependent, leading to growth rates that can be substantially lower than the those predicted by the capacitance model (Nelson and Baker 1996). Only a handful of cloud modeling studies include 
supersaturation-dependent attachment kinetics that are consistent with the theory of faceted growth (MacKenzie and Haynes 1992; Wood et al. 2001; Zhang and Harrington 2015). All other studies assume either perfectly efficient attachment kinetics (capacitance growth) or constant attachment efficiencies (deposition coefficients $\alpha$ ), approximations that are only valid for a narrow range of conditions (Nelson 2005). These simplifications are not limited to the world of model parameterizations, but also appear in interpretations of measurements (Fukuta and Takahashi 1999; Magee et al. 2006).

The ubiquitous use of diffusion-only growth models is driven by the undeniable complexity of crystal growth. However, there has been a trend to develop approximate models that are consistent with the growth of faceted ice. These methods use laboratory-derived parameters to drive changes in particle shape (Chen and Lamb 1994) and to estimate the attachment efficiencies that control mass growth and shape evolution (Wood et al. 2001; Zhang and Harrington 2014). The models are simple enough that they are amenable to application within cloud models, providing a simplified theoretical approach for treating the influences of attachment kinetics on the overall mass growth rate and the evolution of the habits of single crystalline ice (cf. Zhang and Harrington 2015). Moreover, these methods can also be used to extract approximate estimates of attachment kinetic influences on vapor growth from laboratory measurements, thus directly linking laboratory measurements with model parameterizations. In this paper, we provide a composite dataset of characteristic supersaturations $s_{\text {char }}$ that are needed for supersaturationdependent models of $\alpha$. Ledge nucleation has been proposed as the mechanism by which snow grows in atmospheric clouds (Nelson and Knight 1998; Libbrecht 2003b), but this hypothesis has never been explored over the complete tropospheric temperature range with a cloud model. We show that the model of Zhang and Harrington (2014), referred to herein as the Diffusion Surface Kinetics Ice Crystal Evolution (DiSKICE) model, can also reproduce the growth of single crystals at low pressures, and at high (liquid) supersaturations. We also critique existing growth hypotheses that are used to evolve crystal shape (Chen and Lamb 1994; Nelson and Baker 1996). We first review attachment kinetics and vapor growth since these are integral to our analysis.

\section{Ice crystal vapor growth and simplified models}

The rate of vapor uptake by growing crystals depends on the link between surface attachment processes and vapor diffusion. Vapor molecules that impinge upon the surface must find suitable attachment sites before they can incorporate into the bulk crystalline lattice. If suitable attachment sites are uncommon, a surface supersaturation $s_{\text {surf }}$ will develop immediately above the growing surface. Diffusion through the background gas supplies vapor to the growing particle and removes the thermal energy generated by bond formation. The rates of diffusion are driven by vapor and thermal gradients between the surface and the ambient environment, and are therefore inextricably linked to the surface attachment rates. The mass growth of crystals is therefore limited by both diffusion and attachment kinetic processes, and is referred to as diffusion-kinetics limited growth.

\section{a. Surface processes and the deposition coefficients}

During growth, a number of physical processes occur on the crystal surface that ultimately determine the axis and mass growth rates. Vapor molecules must first adsorb to the crystal surface, though not all molecules will necessarily do so. The efficiency of adsorption is often referred to as a "sticking" probability $\alpha_{s}$, and though it is thought that this quantity is near unity (Lamb and Scott 1974; Lamb and Chen 1995; Nelson 2001) at least one experiment suggests it may be quite low (Asakawa et al. 2014). Adsorbed water molecules will migrate across the surface and will desorb unless they find a suitable attachment site such as a ledge or a surface vacancy. At relatively high temperatures $\left(>-5^{\circ} \mathrm{C}\right)$, the surface becomes rough on the growth (nanometer) scale and many attachment sites are available for impinging water molecules (Elbaum 1991). A quasi-liquid layer exists on ice surfaces (Bartels-Rausch et al. 2014) and measurements show occurrences of this layer to temperatures as low as $-30^{\circ} \mathrm{C}$ (Constantin et al. 2018). The existence of this layer has been used in theories of habit development (Kuroda and Lacmann 1982). Attachment kinetics can also change in time because of surface transitions: frozen drops can undergo a faceting transition where small, pyramidal facets quickly grow themselves out of existence leaving only slower growing, larger facets (Gonda and Yamazaki 1984). The formation of grain boundaries in polycrystalline ice at low temperatures $\left(<-20^{\circ} \mathrm{C}\right)$ can be a source of dislocations that substantially alter crystal growth (Pedersen et al. 2011). Low temperature ice is often complex in shape, is affected by both cubic and hexagonal forms with stacking faults (Carignano 2007; Kuhs et al. 2012), and with varied surface processes that control the growth. For example, crossed plates grow with dislocations that propagate parallel to the grain boundary while the remaining facets have slow 
growth rates (Furukawa and Kobayashi 1978). Bicrystals can sprout scrolls (Kobayashi et al. 1976) that may then grow by a protrusion mechanism (Nelson and Swanson 2018).

Though the above surface processes control crystal growth rates, we lack the requisite measurements to formulate general quantitative models. Consequently, surface processes are typically treated in an aggregate sense, and with a single parameter for each facet called a deposition coefficient $\alpha$. The deposition coefficient is the probability that a molecule impinging on the surface will contribute to bulk mass and axis growth, and it acts as a growth efficiency. The deposition coefficient has been measured in numerous studies, often with the approximation that $\alpha$ is constant. The measurements have been scattered from low $(\sim 0.001$; Choularton and Latham 1977; Magee et al. 2006) to high ( $>0.2$; Skrotzki et al. 2013; Kong et al. 2014) values. However, treating $\alpha$ as a constant is only valid for a small range of environmental conditions, crystal sizes, and specific, constant surface types.

The only available models of $\alpha$ are valid for faceted growth and they are supersaturation dependent. A parametric model of $\alpha$ was proposed by Nelson and Baker (1996):

$$
\alpha\left(s_{\text {surf }}, T\right)=\alpha_{s}\left(\frac{s_{\text {surf }}}{s_{\text {char }}}\right)^{m} \tanh \left(\frac{s_{\text {char }}}{s_{\text {surf }}}\right)^{m},
$$

where $\alpha_{s}$ is the sticking probability, which is assumed to be unity herein (see above); $m$ is an adjustable parameter; $s_{\text {surf }}$ is the surface supersaturation; and $s_{\text {char }}$ is a laboratory-measured "characteristic" supersaturation. This latter quantity is, effectively, a scaling parameter that controls the supersaturation dependence of $\alpha .{ }^{1}$ The model treats growth by surface ledges in the sense that $\alpha$ rises commensurately with $s_{\text {surf }}$ : as $s_{\text {surf }}$ becomes larger, the density of ledges rises leading to more efficient growth. The transition from inefficient ( $\alpha$ near zero) to efficient growth ( $\alpha$ near unity) is controlled by $s_{\text {char }}$. The parameter $m$ determines the growth mechanism with a value of $m=1$ corresponding to growth by permanent dislocations as originally derived in the pioneering work of Burton et al. (1951), but given in the above form by

\footnotetext{
${ }^{1}$ It is worth noting that this scaling supersaturation is referred to as a "critical" supersaturation in the theory of ledge nucleation [e.g., Nelson 2001; Eq. (1) herein] and as a "transition" supersaturation when growth is controlled by spiral dislocations (Lamb 2000; Magee et al. 2006). However, we avoid using these terms since each of these two quantities has a specific theoretical definition, whereas the scaling supersaturation derived from measurements is often a parametric value.
}

Lamb and Scott [1974, their Eq. (43)]. Values of $m \geq 10$ were shown by Nelson and Baker (1996) to be representative of growth by ledge nucleation. Dislocations are a permanent source of ledges and produce relatively efficient growth even at low $s_{\text {surf }}$ whereas ledge nucleation causes a rapid onset of growth when $s_{\text {surf }}$ is near $s_{\text {char }}$. Growth is not strongly dependent on $m$ once the value is larger than 10 (see Zhang and Harrington 2015, their Fig. 1). In the studies below, we use $m=1$ for dislocation growth and $m=10$ for ledge nucleation as these are thought to be the primary growth mechanisms for faceted ice.

\section{b. Diffusion-kinetics limited growth model}

Including $\alpha$ [Eq. (1)] in calculations of the mass and axis growth rates requires a model for the gas-phase diffusion of vapor and thermal energy. While methods exist for explicitly solving the diffusion-kinetic growth problem for faceted single crystals (Nelson and Baker 1996; Wood et al. 2001), these methods are complex and simplified methods rooted in the capacitance model are an attractive alternative. Zhang and Harrington (2014) developed a modified version of the capacitance model that calculates $\alpha$ for the major and minor axes of spheroidal ice crystals, which are used to represent the general shape of atmospheric ice. The semidimensions are defined in relation to the hexagonal structure of single crystalline ice where $a$ is half the basal plane maximum width, and $c$ is half the prism plane height. The model deviates from the capacitance model in that axis-dependent vapor fluxes are used to determine $s_{\text {surf }}$ and, therefore, the deposition coefficients for each axis $\alpha_{a}$ and $\alpha_{c}$ using Eq. (1). This mimics the boundary condition for faceted growth, allowing different vapor densities over the $a$ and $c$ axes and leads to [see Zhang and Harrington (2014) for details]

$$
\frac{d m_{i}}{d t}=4 \pi C(c, a) \rho_{\mathrm{eq}} s_{i}\left[\frac{1}{D_{\mathrm{eff}}}+\frac{\rho_{\mathrm{eq}} l_{s}}{K_{T}^{\prime} T}\left(\frac{l_{s}}{R_{v} T}-1\right)\right]^{-1} .
$$

In the above equation, $m_{i}$ is the crystal mass, $C(c, a)$ is the capacitance [see Westbrook et al. (2008) for comprehensive formulations], $\rho_{\mathrm{eq}}$ is the ice equilibrium vapor density, $s_{i}$ is the ambient ice supersaturation (supersaturation), $R_{v}$ is the water vapor gas constant, $l_{s}$ is the sublimation enthalpy, $T$ is the temperature, and $K_{T}^{\prime}$ is the thermal conductivity. Equation (2) is identical to the capacitance model except that the diffusivity of vapor in air $D_{v}$ is replaced with a modified form $D_{\text {eff }}$ that combines the influences of gas-phase vapor diffusion and attachment kinetics [Zhang and Harrington 2014, their Eq. (11)]: 


$$
D_{\text {eff }}=\frac{2}{3} \frac{D_{v}}{\frac{4 D_{v} C}{\alpha_{a} \bar{v}_{v} a c}+\frac{C}{C_{\Delta}}}+\frac{1}{3} \frac{D_{v}}{\frac{4 D_{v} C}{\alpha_{c} \bar{v}_{v} a^{2}}+\frac{C}{C_{\Delta}}},
$$

where $\bar{v}_{v}$ is the vapor mean molecular speed and $C_{\Delta}=C(a+\Delta, c+\Delta)$ is capacitance evaluated at a distance $\Delta$ (approximately the mean free path) away from the crystal surface. The axis-dependent $\alpha$ values, therefore, enter directly into the mass growth rate through $D_{\text {eff }}$ and are calculated as in Zhang and Harrington (2014). Since the deposition coefficients are usually less than unity, $D_{\text {eff }}$ is less than $D_{v}$ leading to mass growth rates that can be substantially lower than the capacitance model. At high supersaturation $\alpha$ can approach unity, and $D_{\text {eff }}$ then becomes very nearly $D_{v}$. Therefore, the capacitance model is recovered when $\alpha$ is near unity and growth becomes diffusion limited. Since Eqs. (2) and (3) are applicable to the diffusion and attachment kinetics growth limits we refer to it as the DiSKICE model.

\section{c. Aspect-ratio evolution}

Aspect-ratio evolution requires a theory for the distribution of mass along each axis, and the theories of Chen and Lamb (1994) and of Nelson and Baker (1996) are generally used. Chen and Lamb (1994) hypothesized that the ratio of the axis growth rates is directly proportional to the aspect ratio $(\phi=c / a)$ and the ratio of the deposition coefficients $\Gamma$ :

$\frac{d c}{d a}=\frac{\alpha_{c}}{\alpha_{a}} \frac{c}{a}=\Gamma \phi \quad$ (aspect-ratio-based hypothesis).

This hypothesis has been used in the development of habit-evolving microphysical models (Chen and Lamb 1999; Hashino and Tripoli 2007; Chen and Tsai 2016; Jensen et al. 2017), and in the interpretation of laboratory measurements (Sulia and Harrington 2011; Connolly et al. 2012).

Crystals growing by ledge nucleation likely have ledges that form near crystal edges where $s_{\text {surf }}$ is greatest (Frank 1982, see their Fig. 18). In this case, the ratio of the axis growth rates depends only on the deposition coefficients (Nelson and Baker 1996):

$$
\frac{d c}{d a}=\frac{\alpha_{c}}{\alpha_{a}} \quad \text { (facet-based hypothesis) }
$$

This hypothesis is less commonly used in cloud modeling (Wood et al. 2001), but it has been used to interpret laboratory growth data (Nelson and Knight 1998; Nelson 2001). It is worth noting that this hypothesis also breaks down in some situations. Ledge sources can be at locations besides crystal edges (Nelson 2001), crystal hollowing may require a transition from dislocation to ledge nucleation growth (Nelson and Knight 1998), crystals often have nonfaceted "rough" regions that may affect habit development (Pfalzgraff et al. 2010), and molecules may migrate across crystal edges thus influencing ledge nucleation rates (Frank 1982). At this stage, however, there is no theoretical approach for including these more detailed processes in a simplified model of single crystal habit development.

As the above discussion implies, our theoretical knowledge is insufficient to explicitly model the development of secondary habit features that appear at high supersaturations, such as dendritic branching and hollowing. These features are normally treated through an "effective" density $\rho_{\text {eff }}$ that is lower than the bulk density of ice and accounts for the branches and hollowed regions that are not represented in the modeled particle. This approach has a long history in modeling (Miller and Young 1979; Chen and Lamb 1994; Thompson et al. 2008) and in data analysis (Fukuta 1969; Fukuta and Takahashi 1999). Chen and Lamb (1994) approximate the density added during growth (the deposition density) with an empirical equation that depends on temperature and supersaturation [their Eq. (42)]. This form allows the effective density to decline during growth and is used in the simulations presented below.

For the sake of completeness, we note that laboratory evidence suggests the aspect ratio should be treated as a constant during sublimation with a sublimation coefficient of unity (Nelson 1998), an approach we advocate here. This result has a physical basis: measurements suggest that crystal roughening during sublimation (Nelson 1998; Magee et al. 2014) causes the sublimation coefficient to approach unity. As a consequence, the vapor density becomes constant along the surface leading to a constant aspect ratio (shape is preserved) during sublimation (Ham 1959).

\section{Characteristic supersaturations}

\section{a. Synopsis of previously published data}

Characteristic supersaturations are required as input to the $\alpha$-dependent growth model; however, available measurements of $s_{\text {char }}$ (Fig. 1) are sparse and restricted to temperatures above $-40^{\circ} \mathrm{C}$. Various laboratory devices along with different models of $\alpha$ have been used in prior measurements of $s_{\text {char }}$. We therefore expect variations in $s_{\text {char }}$ that are due solely to differences in the measurement techniques and analytical models employed 


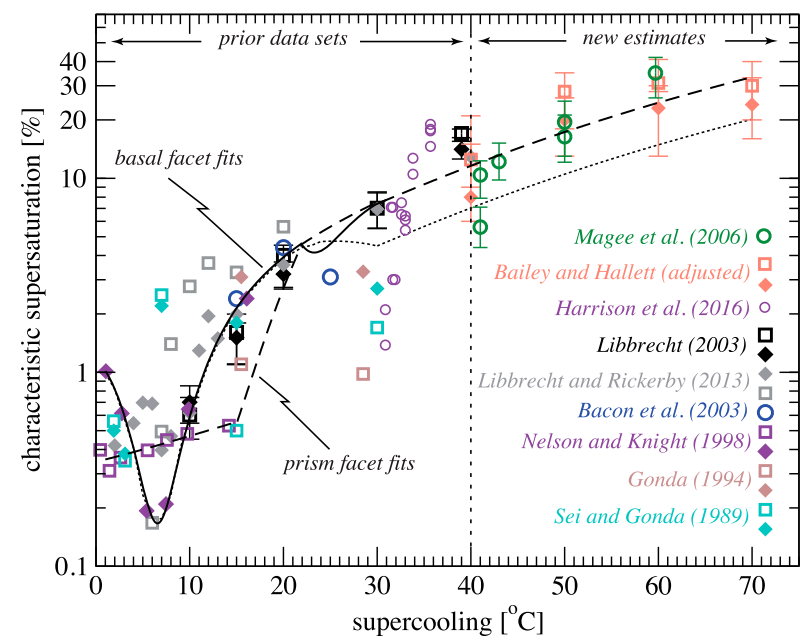

FIG. 1. Characteristic supersaturations as a function of supercooling $\left(\Delta T=T_{o}-T\right)$ from laboratory-measured vapor growth rates (Sei and Gonda 1989; Gonda et al. 1994; Nelson and Knight 1998; Bacon et al. 2003; Libbrecht 2003b; Libbrecht and Rickerby 2013; Harrison et al. 2016). Prior values of $s_{\text {char }}$ exist for temperatures above $-40^{\circ} \mathrm{C}$ only. The dataset is extended to temperatures below $-40^{\circ} \mathrm{C}$ using estimates from the growth data of Magee et al. (2006) and Bailey and Hallett (2010). Values of $s_{\text {char }}$ for the basal and prism facets are denoted by diamond and square symbols, respectively. A circle is used to denote $s_{\text {char }}$ that is representative of the overall particle growth. Symbol color indicates the data source. Best fits to the data using polynomials are indicated by the black line (solid: basal facet; dashed: prism facet). The black dotted curve is an ad hoc modification of the basal $s_{\text {char }}$ to produce columnar growth at $T<-20^{\circ} \mathrm{C}$.

in a given study. It is beyond the scope of this work to assess and interpret these differences; instead, we have collected prior published data and use them to provide a proxy dataset.

The available data clearly indicate that $s_{\text {char }}$ increases commensurately with the supercooling $\left(T_{o}-T\right.$; $T_{o}=273.15 \mathrm{~K}$; Fig. 1). Values of $s_{\text {char }}$ are relatively low $(<2 \%)$ at temperatures above $-20^{\circ} \mathrm{C}$ and their variation with temperature is consistent with the primary habits of ice for most of the datasets: At a given temperature, the major growth axis has lower values of $s_{\text {char }}$ than the minor axis, and this difference will produce a larger $\alpha$ in Eq. (1) and faster major axis growth. At temperatures above $-20^{\circ} \mathrm{C}$ the data of Libbrecht and Rickerby (2013) generally have the highest $s_{\text {char }}$ and these data show basal and prism values for $s_{\text {char }}$ that are the opposite of the other published measurements. Libbrecht and Rickerby (2013) point out that this result may be due to processes occurring on the crystal surface that are not captured by the standard models of $\alpha$. Nevertheless, using these data directly in Eq. (1) produces the wrong primary habits and they are not included in our studies above $-20^{\circ} \mathrm{C}$.
Values of $s_{\text {char }}$ become progressively larger as the temperature falls below $-20^{\circ} \mathrm{C}$ indicating that growth becomes more strongly limited by attachment kinetics. The datasets are relatively consistent with one another though substantial outliers occur near $-30^{\circ} \mathrm{C}$. Only two published measurements of the basal and prism facet $s_{\text {char }}$ exist, and they are consistent with one another below $-20^{\circ} \mathrm{C}$ (Libbrecht 2003b; Libbrecht and Rickerby 2013). The measured $s_{\text {char }}$ are nearly the same on the basal and prism facets, with habit development controlled by the leading coefficient in the ledge nucleationrate equation (Libbrecht 2003b). These results contrast with those of (Nelson and Knight 1998) in which the primary habits are controlled by the difference in $s_{\text {char }}$ between the basal and prism facets at higher temperatures. Zhang and Harrington (2014) used the data of Libbrecht (2003b) to derive values of $s_{\text {char }}$ consistent with the formulation of Nelson and Knight (1998); however, these derived values produce thick columns and plates at high supersaturation, whereas thinner particles are often observed. Libbrecht (2003a) has hypothesized that the production of thinner plates may be due to structure-dependent $\alpha$ that is currently not accounted for in theories. Unfortunately, there is no consensus on the modeling of the primary habits at temperatures below $-20^{\circ} \mathrm{C}$, though the values of $s_{\text {char }}$ are relatively consistent among the datasets. At lower temperatures, we therefore estimate the reduction in the mass growth rate by using a single, particle-averaged value of $s_{\text {char }}$ following Zhang and Harrington (2014).

The use of a single $s_{\text {char }}$ to characterize attachment kinetic influences on growth has precedence. Our prior work (Zhang and Harrington 2014, Fig. 10 therein) showed that the mass uptake of nonspherical single crystals is very nearly reproduced if a particle-average value of $s_{\text {char }}$ and an equivalent volume sphere are used in the mass growth calculations. Figure 2 shows a similar result for the instantaneous mass growth rate (normalized to the capacitance rate) for a large range of aspect ratios, supersaturations, and for both ledge nucleation and dislocation growth. The relative errors between the solutions using separate $s_{\text {char }}$ for each axis, and the solutions using an average $s_{\text {char }}$, are generally less than $5 \%$; however, the relative error becomes substantial $(20 \%$ or larger $)$ when crystals are small $(r<$ $10 \mu \mathrm{m}$ ) and the supersaturation is near $s_{\text {char }}$ (not shown). Given that an average value of $s_{\text {char }}$ and an equivalent volume sphere provide an accurate estimate of the overall mass growth rate for highly anisotropic singlecrystal growth, we hypothesize that it is reasonable to employ this method for the growth of crystals with more complex facet morphologies, as is observed near liquid saturation and in polycrystalline ice. 


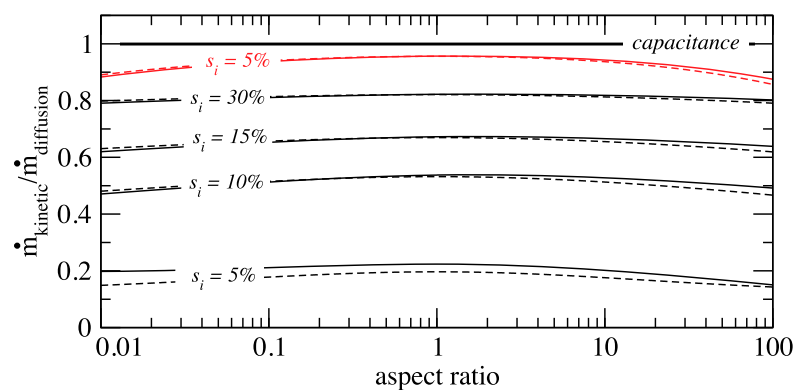

FIG. 2. Ratio of diffusion-kinetics limited growth rate to the maximum (capacitance) growth rate as a function of the aspect ratio for a major crystal axis length of $200 \mu \mathrm{m}$. A temperature of $-35^{\circ} \mathrm{C}$ and pressure of $500 \mathrm{hPa}$ were used. The solid lines used an $s_{\text {char }}$ of $11 \%$ and $5.5 \%$ for the major and minor crystal axes, respectively. The dashed lines used an average $s_{\text {char }}$ and assumed an equivalent volume spherical crystal. Black curves indicate ledge nucleation whereas the red curves indicates dislocation growth at $s_{i}=5 \%$.

This hypothesis underlies the estimates of $s_{\text {char }}$ provided in the following subsection.

\section{b. Estimating characteristic supersaturations at $\mathrm{T}<-40^{\circ} \mathrm{C}$}

To our knowledge, no data exist for $s_{\text {char }}$ and the growth rates of individual crystal facets at temperatures below $-40^{\circ} \mathrm{C}$. However, mass growth rate data do exist at these temperatures for individually grown crystals (Magee et al. 2006). These growth data are particularly useful as they record the vapor growth and sublimation of individual crystals, formed from frozen small droplets (radius of 5-7 $\mu \mathrm{m}$ ), at nearly constant temperatures of $-59.8^{\circ},-50^{\circ},-44^{\circ}$, and $-42^{\circ} \mathrm{C}$. Estimates of $\alpha$ were low $(\simeq 0.006 \pm 0.002)$ and different values of $\alpha$ were required to fit each growth and sublimation portion of the time series (Magee et al. 2006). Crystal growth was likely inhibited by attachment kinetics indicating that it would be useful to estimate an particle-average value of $s_{\text {char }}$ from the data.

An example of the growth data is shown in Fig. 3a for a crystal undergoing cycles of sublimation and growth at a temperature of $-59.8^{\circ} \mathrm{C}$ and a pressure of $972 \mathrm{hPa}$. The mass evolution of the crystal depends on its initial size, which can be determined to about $1 \mu \mathrm{m}$. This size uncertainty dominates the errors in determining $\alpha$ and therefore $s_{\text {char }}$, whereas supersaturation errors have a smaller influence on $\alpha$ (Magee et al. 2006). We use the DiSKICE model to fit the measured mass ratio time series by minimizing on a value of $s_{\text {char }}$. We assume spherical particles because the grown crystals were small (5 to $20 \mu \mathrm{m}$ in radius), and therefore had likely not developed pronounced habits. In the model we also assume that the crystals grow by ledge nucleation. Equally
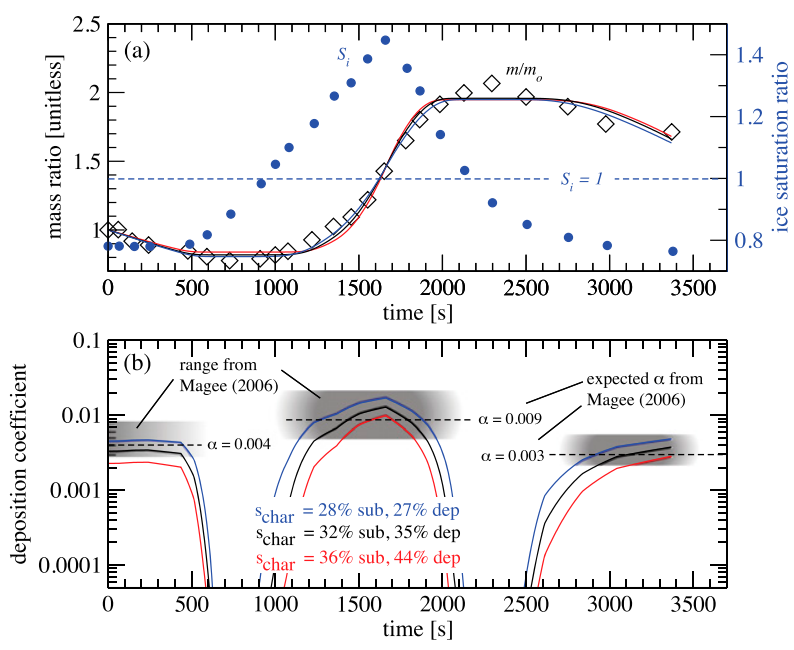

FIG. 3. Evolution of (a) the measured mass ratio and ice saturation ratio and (b) the deposition coefficient at $-59.8^{\circ} \mathrm{C}$ and $972 \mathrm{hPa}$. The measured mass-ratio $\left(\mathrm{m} / \mathrm{m}_{o}\right.$ where $m_{o}$ is the initial mass) is given by the black diamonds and ice saturation ratio $S_{i}$ is given by the blue circles. Best fits to the mass-ratio time series using the DiSKICE model are shown by the solid lines. The fits used the most likely initial radius (black line; $r_{o}=7 \mu \mathrm{m}$ ) along with the upper (blue line; $r_{o}=8.4 \mu \mathrm{m}$ ) and lower (red line; $r_{o}=5.4 \mu \mathrm{m}$ ) limits, since uncertainty in the initial radius dominates the error. Evolution of the predicted deposition coefficient uses the three initial radii given in (a). Also shown are the best estimate $\alpha$ (black dashed lines and numbers) and the range (shaded region) from Magee (2006). Best-fit values of $s_{\text {char }}$ for sublimation (sub) and deposition (dep) from each DiSKICE time series are given on the figure and color-coded to match the simulations.

accurate fits are possible if dislocation growth is assumed; however, a low sticking efficiency $\left(\alpha_{s} \sim 0.004\right)$ is then required. Though this result is conceivable, it seems unlikely as high values of $\alpha$ (above 0.1 ) that have been estimated at $T<-40^{\circ} \mathrm{C}$ (Skrotzki et al. 2013; Pokrifka 2018) would then not be possible even for dislocation growth at liquid saturation.

The model fits to the measured mass ratios are shown in Fig. 3a for the most probable initial radius $\left(r_{o}=7 \mu \mathrm{m}\right)$ and the upper $\left(r_{o}=8.4 \mu \mathrm{m}\right)$ and lower $\left(r_{o}=5.4 \mu \mathrm{m}\right)$ uncertainty bounds. The fit has the same accuracy as that of Magee et al. (2006) except that $\alpha$ varies with time (Fig. 3b), rising and decreasing commensurately with the supersaturation. The rapid decline in $\alpha$ with decreasing supersaturation is the reason the model captures the relatively flat region in the mass growth time series (such as $500-1000 \mathrm{~s}$ ) that is not reproducible with a diffusionlimited growth model. More critically, the values of $s_{\text {char }}$ (in Fig. 3b) required to fit each growth and sublimation period are relatively similar to one another. These results suggest that a similar surface process is occurring on these crystals throughout the growth cycle. Finally, note that the predicted time series of $\alpha$ falls within the 
range (gray shades) determined by Magee (2006) with $\alpha$ that are similar to his fitted values (dashed lines).

The values of $s_{\text {char }}$ determined from the above growth time series, and the other experiments at $-50^{\circ},-44^{\circ}$, and $-42^{\circ} \mathrm{C}$ (not shown), are given in Fig. 1. Note that values for the sublimation portions of the time series are not shown since the present study focuses on depositional growth. The values of $s_{\text {char }}$ determined from the fitting procedure are somewhat lower than prior measurements near $-40^{\circ} \mathrm{C}$, but show a similar temperature trend for $s_{\text {char }}$ as the higher temperature data. The approximate consistency of our results with prior data is encouraging, but comparisons with independent data would be useful. In particular, experiments run over a range of supersaturation for fixed temperatures and single crystalline ice would be ideal.

It is important to point out that one should exercise caution in the use of the estimates of $s_{\text {char }}$ provided herein since it is always possible that experimental artifacts affected the resulting growth curves. It is also not known whether the measured crystals developed facets quickly after the periods of sublimation, though the observations of Gonda and Yamazaki (1978) suggest that facets appear rapidly after droplet freezing and the electron microscope studies of Pfalzgraff et al. (2010) show that facets rapidly reappear (within $90 \mathrm{~s}$ to a few minutes) during regrowth following sublimation. In addition, Magee et al. (2006) found that low values of $\alpha$ were required for sublimation, a result that is inconsistent with other measurements (Nelson 1998; Magee et al. 2011).

\section{c. Comparisons with effective capacitance measurements}

Bailey and Hallett (2004) reported on thermal gradient diffusion chamber measurements of crystals grown on a substrate. From these growth measurements capacitance values normalized to the maximum dimension $L_{i}$ were extracted for hexagonal plates and columns (Bailey and Hallett 2010). These values were estimated by using the measured mass growth rate and then solving for the capacitance in the capacitance mass growth model. The normalized capacitance is a useful metric for vapor growth since it is independent of size, and depends only on the aspect ratio for diffusion-limited growth. Normalized capacitance values extracted from growth data would therefore fall approximately along the solid black lines in Fig. 4 if growth is diffusion limited. However, Bailey and Hallett (2010) found that the measured normalized capacitance was substantially lower than capacitance theory (Bailey and Hallett 2010, their Fig. 12). Their data span a relatively large range of $L_{i}(50-$ $400 \mu \mathrm{m})$, pressure $p(500-150 \mathrm{hPa})$, and supersaturation $s_{i}\left(1 \%-13 \%\right.$ at $-40^{\circ} \mathrm{C}$, and $5 \%-25 \%$ at lower temperatures), and an apparent aspect-ratio dependence reminiscent of capacitance theory appears in the data. The approximate ranges of their data are shown in Fig. 4.

There are many possible reasons why the extracted values of the normalized capacitance are lower than capacitance theory (see Bailey and Hallett 2010), but one main reason is that attachment kinetics are not included in the capacitance model. Therefore, the extracted values of $C / L_{i}$ are convolved with the attachment kinetics, producing an effective normalized capacitance $c_{\text {eff }}$ that can be computed with DiSKICE. Values of $c_{\text {eff }}$ are computed using DiSKICE assuming ledge nucleation growth and using values of $s_{i}, L_{i}$, and $p$ in the middle of the measured range. The spread of model solutions is calculated using the range of measured $s_{i}$ (light shades) and $L_{i}$ and $p$ (dark shades). The calculated $c_{\text {eff }}$ using the $s_{\text {char }}$ values derived in section $2 \mathrm{~b}$ (green lines, Fig. 4) are often consistent with the range of the measurements, though at $-40^{\circ}$ and $-50^{\circ} \mathrm{C}$ the calculated values are too high for planar crystals and at $-60^{\circ} \mathrm{C}$ the calculated values are at the lower end of the measured range. Including the variability in $s_{i}, L_{i}$, and $p$ produces a range of solutions that has a spread similar to the red and blue shaded regions (discussed below) and often encompasses the measured range (omitted for clarity).

Because some of the $c_{\text {eff }}$ calculations fall outside of the observed range, it is useful to calculate adjusted values of $s_{\text {char }}$ so that spread in the solutions is confined primarily within the measured range of $c_{\text {eff }}$. This was done by adjusting $s_{\text {char }}$ until the accumulated error in the spread of the model solutions as compared to the observed range of $c_{\text {eff }}$ is a minimum. These are shown as the red and blue shaded regions in Fig. 4 along with model solutions using the midrange values of $s_{i}, L_{i}$, and $p$ (solid red and blue lines). The spread in the solutions for $c_{\text {eff }}$ is weakly dependent on $p$ and $L_{i}$, but is dominated by $s_{i}$ with a spread that is similar to that of the measured range; these are expected results since attachment kinetics are dominated by the supersaturation dependence through $\alpha$. The adjusted values of $s_{\text {char }}$, along with a large uncertainty based on the spread of $s_{i}$ values, are given in Fig. 4 and Fig. 1. These adjusted values are generally consistent with those from the data of Magee et al. (2006), and also provide a data point at $-70^{\circ} \mathrm{C}$.

Naturally, one should bear in mind that these adjusted values of $s_{\text {char }}$ are very rough estimates calculated from measured growth rates of crystals of various sizes over a range of pressures and supersaturations. Furthermore, since numerous crystals were grown from the substrate 

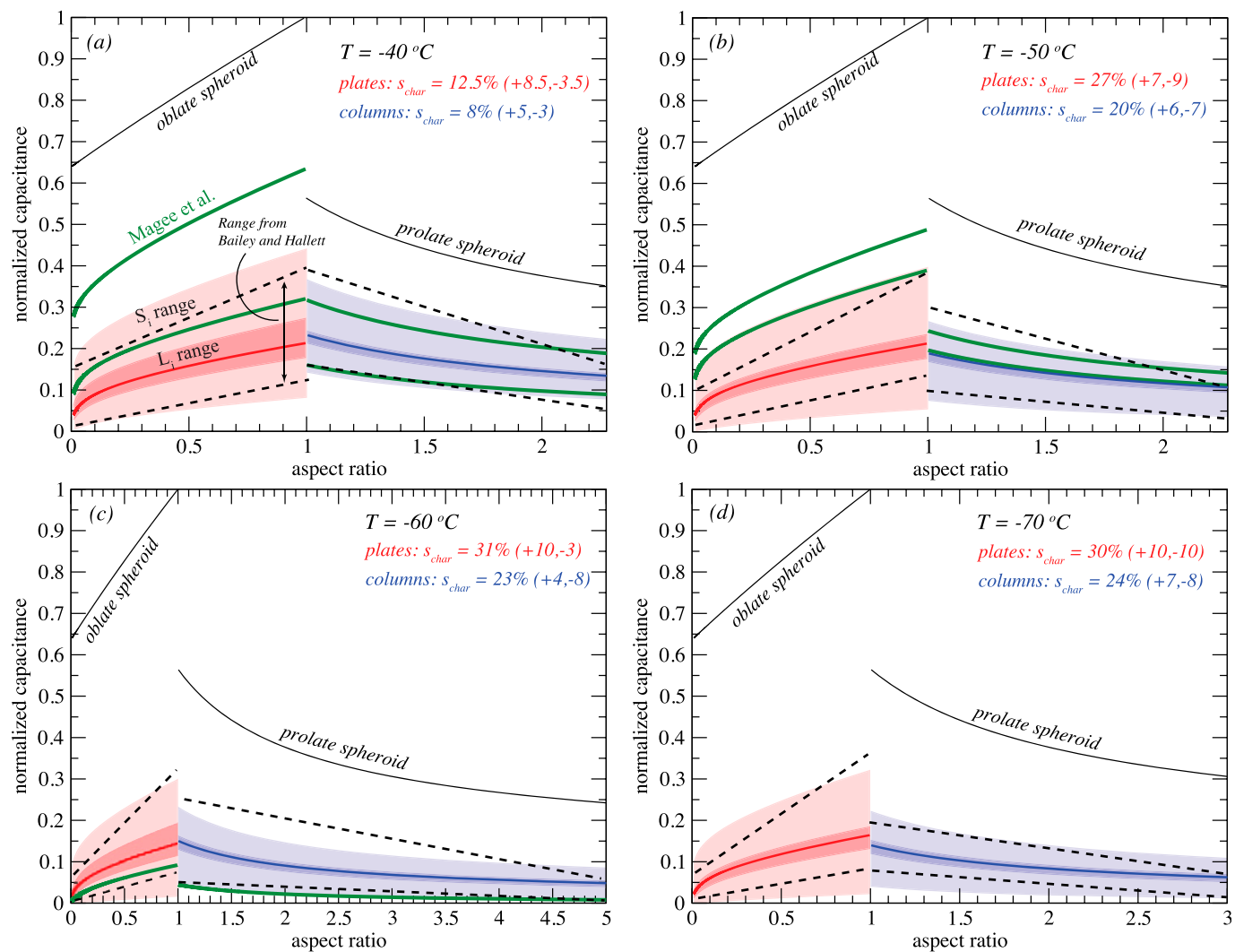

FIG. 4. Normalized effective capacitance [ $C / a$ for plates and $C /(2 c)$ for columns] as a function of aspect ratio $\phi$ at temperatures between $-40^{\circ}$ and $-70^{\circ} \mathrm{C}$. The solid black lines are capacitance model results for oblate and prolate spheroids. Black dashed lines indicate the approximate range of measured values given in Bailey and Hallett (2010). Green lines used $s_{\text {char }}$ values (two at $-40^{\circ}$ and $-50^{\circ} \mathrm{C}$ ) from the data of Magee et al. (2006). Calculations used midrange values of crystal maximum length $L_{i}$ of $200 \mu \mathrm{m}$, a pressure $p$ of $300 \mathrm{hPa}$, and ice supersaturations $s_{i}$ of $7 \%$, $10 \%, 15 \%$, and $15 \%$ for temperatures of $-40^{\circ},-50^{\circ},-60^{\circ}$, and $-70^{\circ} \mathrm{C}$, respectively. Adjusted solutions using these midrange values and the DiSKICE model for plates (columns) are indicated by the thick red (blue) lines. Darkshaded regions indicate the variability due to the range of $L_{i}(50-400 \mu \mathrm{m})$ and $p(500-150 \mathrm{hPa})$. Light-shaded regions indicate the variability due to the range of $s_{i}$ (generally $5 \%-25 \%$ ). Variability ranges are similar for the green curves (not shown for clarity). Adjusted values of $s_{\text {char }}$ and the variability range (located in the upper right of each panel) are colored to match the lines.

in these experiments, the vapor diffusion field in the chamber deviated from that of a classic flat-plate diffusion chamber. The simulations of Westbrook et al. (2008) show that crystal crowding could have reduced the measured growth rates by as much as a factor of 3 , though Bailey and Hallett (2010) point out that they only analyzed data from conditions that were not crowded (their Fig. 11). Though Bailey and Hallett (2010) evidently took great care to exclude crowded conditions, the influence of the population of crystals on the three-dimensional vapor field, and the horizontal diffusion that must occur, is not known. Moreover, as a reviewer of the current paper pointed out, the crystals grown from the central strand are asymmetric since one end is attached to the substrate. The attached end of the crystal therefore cannot grow naturally and deplete the vapor supply, and it is unknown how important this effect would be to the measured growth rates.

\section{d. Polynomial fits to characteristic supersaturation data}

A subset of the data shown in Fig. 1 is used to produce polynomial fits (Table 1) to $s_{\text {char }}$ as a function of temperature (black curves). The data subsets used in the fits were selected as follows: At temperatures above $-20^{\circ} \mathrm{C}$, the data from Nelson and Knight (1998) and Libbrecht (2003b) were used. The data from Libbrecht and Rickerby (2013) were not used for reasons discussed earlier, and Sei and Gonda (1989) was excluded because the growth was possibly influenced by thermal substrate effects (Nelson 1993). At temperatures below $-20^{\circ} \mathrm{C}$ the data of Libbrecht (2003b) are used along with 
TABLE 1. Polynomial fits to $s_{\text {char }}$ [used in Eq. (1)] as a function of temperature where $\Delta T=T-T_{o}$ and $T_{o}=273.15 \mathrm{~K}$ for the $c$ axis (basal facet) and $a$ axis (prism facet).

\begin{tabular}{|c|c|c|c|c|c|c|c|}
\hline Temperature & $a_{0}$ & $a_{1}$ & $a_{2}$ & $a_{3}$ & $a_{4}$ & $a_{5}$ & $a_{6}$ \\
\hline \multicolumn{8}{|c|}{$c$-axis fit coefficients: $s_{\text {char }}=\sum_{n=0}^{6} a_{n} \Delta T^{n}$} \\
\hline$-22^{\circ}<T \leq-1^{\circ} \mathrm{C}$ & 1.1217 & 0.0381 & -0.08375 & -0.01573 & -0.001011 & $-2.915 \times 10^{-5}$ & $-3.182 \times 10^{-7}$ \\
\hline$-30^{\circ} \leq T \leq-22^{\circ} \mathrm{C}$ & 753.63 & 105.97 & 5.553 & 0.1281 & 0.0011 & 0 & 0 \\
\hline$T<-30^{\circ} \mathrm{C}$ & 3.7955 & 0.10614 & 0.00753 & 0 & 0 & 0 & 0 \\
\hline \multicolumn{8}{|c|}{$a$-axis fit coefficients: $s_{\text {char }}=\sum_{n=0}^{6} a_{n} \Delta T^{n}$} \\
\hline$-15^{\circ}<T \leq-1^{\circ} \mathrm{C}$ & 0.3457 & -0.0093 & 0.000308 & 0 & 0 & 0 & 0 \\
\hline$-22^{\circ}<T \leq-15^{\circ} \mathrm{C}$ & -5.2367 & -1.3184 & -0.1107 & -0.00323 & 0 & 0 & 0 \\
\hline$-30^{\circ} \leq T \leq-22^{\circ} \mathrm{C}$ & -0.7106 & -0.1478 & 0.00423 & 0 & 0 & 0 & 0 \\
\hline$T<-30^{\circ} \mathrm{C}$ & 3.7955 & 0.10614 & 0.00753 & 0 & 0 & 0 & 0 \\
\hline
\end{tabular}

$s_{\text {char }}$ estimated in sections $2 \mathrm{~b}$ and $2 \mathrm{c}$ above. The basal facet $s_{\text {char }}$ for temperatures between $-20^{\circ}$ and $-30^{\circ} \mathrm{C}$ (black solid curve) is taken from the estimates made by Wood et al. (2001). We use a particle-average $s_{\text {char }}$ and an equivalent volume sphere at temperatures below $-30^{\circ} \mathrm{C}$, since these values are rough approximations of attachment kinetic influences on crystal growth. The only exceptions are model tests of columnar growth for which an ad hoc reduction in the basal $s_{\text {char }}$ is applied (black dotted curve) based on the analysis of Zhang and Harrington (2014).

\section{Single crystal evolution at low and high supersaturation}

The evolution of the primary habits of single crystalline ice depends on the growth hypothesis [Eq. (4) or (5)] that is employed. A number of studies have shown that the aspect-ratio-based hypothesis of Chen and Lamb (1994) can reproduce the evolution of the primary habits of ice at liquid saturation; however, those works were predicated on the assumption that the ratio of the deposition coefficients $\Gamma$ is a constant at a given temperature. It is not immediately evident which hypothesis [Eq. (4) or (5)] provides a more accurate representation of aspect-ratio evolution when deposition coefficients are predicted. Moreover, it is not clear whether ledge nucleation or dislocation growth provides a better representation of axis evolution at high supersaturations. Below, we analyze the two growth hypotheses and extend the studies to secondary habits (branching and hollowing) through a reduced density.

\section{a. Assessment of axis growth hypotheses}

The hexagonal ice growth model developed by Wood et al. (2001) was used in prior work to assess the axisdependent growth of crystals using DiSKICE (Zhang and Harrington 2014). The hexagonal model solves the
Laplace equation on a triangular grid covering the basal and prism facets of hexagonal ice using the constant-flux boundary condition for faceted growth. The model is limited in that simulations of branched and hollowed crystals are not possible. Nevertheless, the hexagonal model reproduces the general features of faceted growth and provides a convenient comparison basis for simplified theories. For the simulations below, the hexagonal model is set up as in Zhang and Harrington (2014) with ledge nucleation growth occurring where $s_{\text {surf }}$ is a maximum and dislocation growth at the facet centers. Characteristic supersaturations used in DiSKICE and the hexagonal model were calculated from the polynomial fits in Fig. 1, though the short dashed curve is used for the basal facets at temperatures below $-20^{\circ} \mathrm{C}$ so that a columnar aspect ratio develops (discussed below).

Results of the comparison between the hexagonal and DiSKICE model in the work of Zhang and Harrington (2014) were encouraging in the sense that the general dependence of growth on aspect ratio and $\alpha$ were captured by the DiSKICE model. However, those studies were limited in a number of ways: they only examined growth by dislocations at high $s_{i}$ and ledge nucleation growth at low $s_{i}$, but did not examine the facetbased growth hypothesis [Eq. (5)], nor growth where branching and hollowing are treated through a reduction in the particle density. While the aspect-ratio-based hypothesis was found to compare well to the hexagonal model, there were indications that dislocations produced excessive columnar growth near $-7^{\circ} \mathrm{C}$. This excessive growth is inherent in the aspect-ratio-based hypothesis, which becomes markedly clear for ledge nucleation growth as is shown in Fig. 5a. After 10 min of growth at liquid saturation with the aspect-ratiobased hypothesis, columnar crystals at temperatures between $-5^{\circ}$ and $-9^{\circ} \mathrm{C}$ are nearly an order of magnitude longer, and significantly thinner, than the 

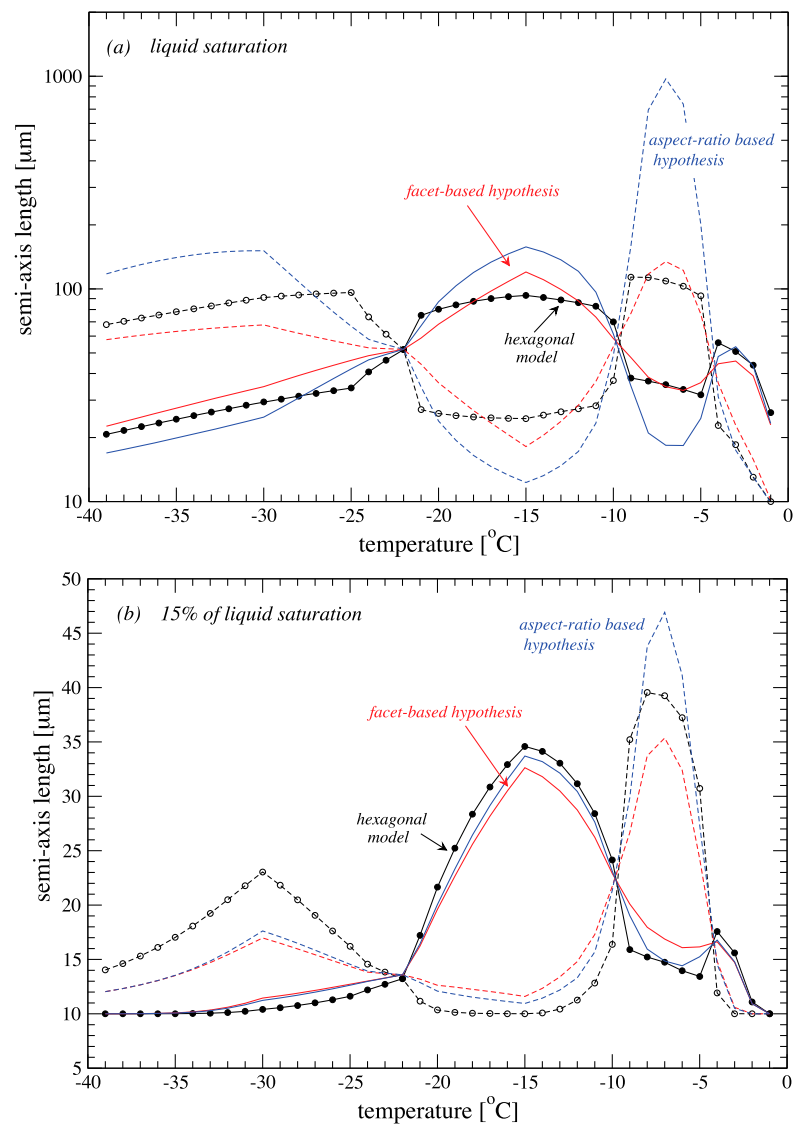

FIG. 5. Comparison of simulated semiaxis lengths from the DiSKICE and hexagonal models, assuming ledge nucleation, after 10 min of growth at (a) high (liquid) saturation and (b) low saturation ( $15 \%$ of the ice saturation ratio at liquid saturation). The $a$-axis length is given by the solid lines and the $c$ axis by the dashed lines. Black lines with circles indicate the hexagonal model solutions, and red and blue lines indicate DiSKICE solutions with the facet-based and aspect-ratio-based hypotheses, respectively.

hexagonal model solutions. This result also occurs at temperatures below $-20^{\circ} \mathrm{C}$; however, the excessive growth is weaker here because of the larger values of $s_{\text {char }}$. Aspect-ratio evolution is also excessive for planar crystals, and so it appears that at high supersaturation the aspect-ratio-based hypothesis produces crystals that are too large and with extreme aspect ratios. This excessive growth is substantially muted at low supersaturations, and only an indication is seen near $-7^{\circ} \mathrm{C}$ (Fig. 5b). In contrast, the facet-based hypothesis produces a much better match to the hexagonal model at high supersaturations, though at low supersaturations there is less of a distinction between the two hypotheses. Growth by dislocations produces a similar result at high (liquid) saturation (Fig. 6) and low saturation (not shown), though the enhancement of axis growth is far lower than it is for ledge nucleation.

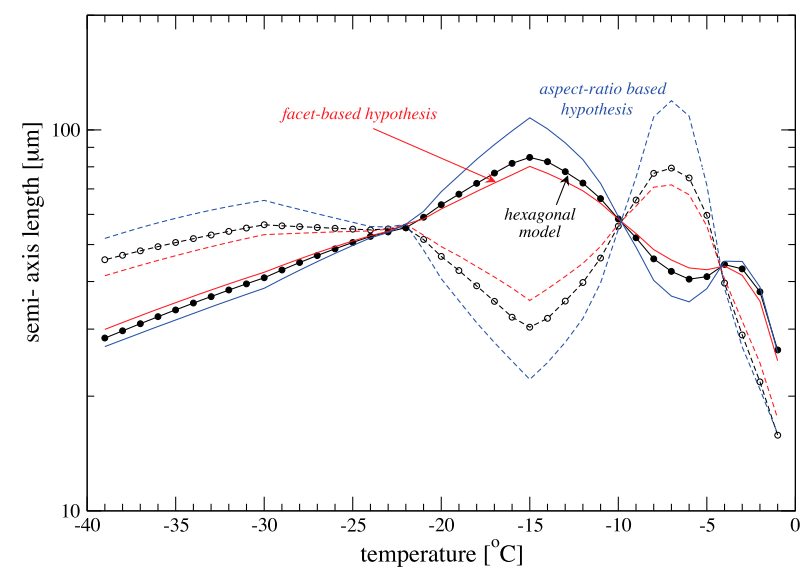

FIG. 6. Comparison of simulated semiaxis lengths from the DiSKICE and hexagonal models, assuming dislocations, after $10 \mathrm{~min}$ of growth at liquid saturation. The $a$ axis is given by the solid lines and the $c$ axis by the dashed lines. Black lines with circles indicate the hexagonal model solutions, and red and blue lines indicate DiSKICE solutions with the facet-based and aspect-ratiobased hypotheses, respectively.

The reasons for enhanced growth in the aspect-ratiobased hypothesis can be understood by examining the time evolution of the semiaxis lengths $\alpha$ and the axisdependent vapor fluxes at $-7^{\circ} \mathrm{C}$, where enhanced growth is the most excessive. The evolution of the $c$ and $a$ axes is clearly better represented by the facet-based hypothesis at all supersaturations (Figs. 7a and 7b). In contrast, the aspect-ratio-based hypothesis becomes progressively worse at higher supersaturations, with time-dependent values of $a$ and $c$ diverging substantially from the hexagonal model solution. At high supersaturation $\left(s_{i}=7 \%\right)$, runaway growth is produced for the $c$ axis while the $a$ axis essentially ceases growth after about $200 \mathrm{~s}$, producing columns with extreme aspect ratios $(\phi=190)$.

Strong growth along the $c$ axis in the aspect-ratiobased hypothesis indicates that $\alpha$ is large (Fig. 8). While the facet-based hypothesis produces $\alpha$ values that follow a similar functional form to those predicted by the hexagonal model, with $\alpha$ for each axis decreasing in time, the aspect-ratio-based hypothesis produces a rise in $\alpha$ for the $c$ axis that is accentuated at higher supersaturations. Interestingly, $\alpha$ for the $a$ axis follows a similar functional form to the hexagonal model solution. The increasing values of $\alpha$ with time indicate that $s_{\text {surf }}$, and hence the vapor flux onto the $c$ axis, must be rising unrealistically in time, which is indeed the case (Fig. 9). This increase in the vapor flux onto the $c$ axis is driven by the aspect-ratio dependence of the growth hypothesis [Eq. (4)]. The equation originates from the ratio of the vapor fluxes along the $c$ and $a$ axes in the capacitance model [Chen and Lamb 1994, their Eq. (25)], and 

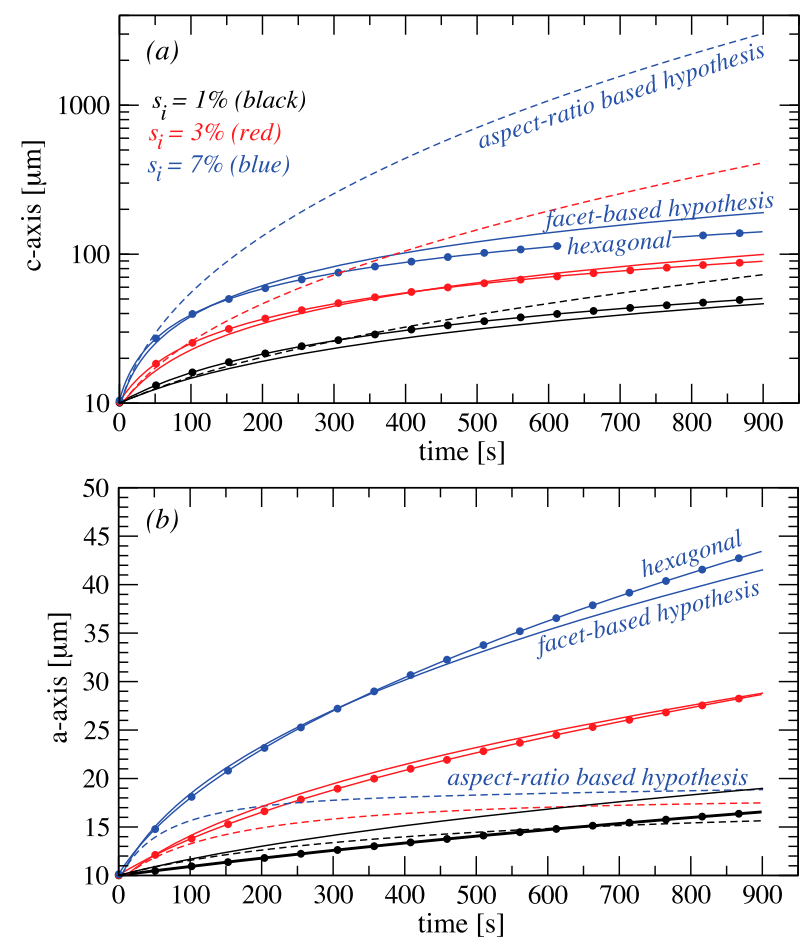

FIG. 7. Time series of (a) $c$-axis and (b) $a$-axis lengths for the simulations shown in Fig. 5; three different ice supersaturations (colored commensurately with lines) are shown at $T=-7^{\circ} \mathrm{C}$. Lines with circles indicate hexagonal model solutions, and solid and dashed lines indicate DiSKICE solutions with the facet-based and aspect-ratio-based hypotheses, respectively.

therefore, the inclusion of $\phi$ essentially multiplies the vapor flux from the facet-based hypothesis by the aspect ratio, causing an increase in the vapor flux by a factor of $\phi$. When $\alpha$ values are predicted, including this scaling by $\phi$ causes a positive feedback where increasing aspect ratio amplifies the fluxes onto the major axis. This result does not occur in the original model of Chen and Lamb (1994) because the ratio of the deposition coefficients is constant at a given temperature. The artificial feedback with aspect-ratio evolution that occurs when $\alpha$ is predicted is a general feature of the aspect-ratio-based hypothesis, and it indicates a flaw in the hypothesis that becomes progressively worse at higher supersaturations. Consequently, models that evolve crystal shapes based on this hypothesis (Hashino and Tripoli 2007; Harrington et al. 2013a; Chen and Tsai 2016) would have to be modified to use the facet-based hypothesis if $\alpha$ is predicted.

\section{b. Comparison with laboratory measurements at liquid saturation}

While the above comparisons, and the studies of Zhang and Harrington (2014), indicate that DiSKICE provides a suitable approximation for single crystal
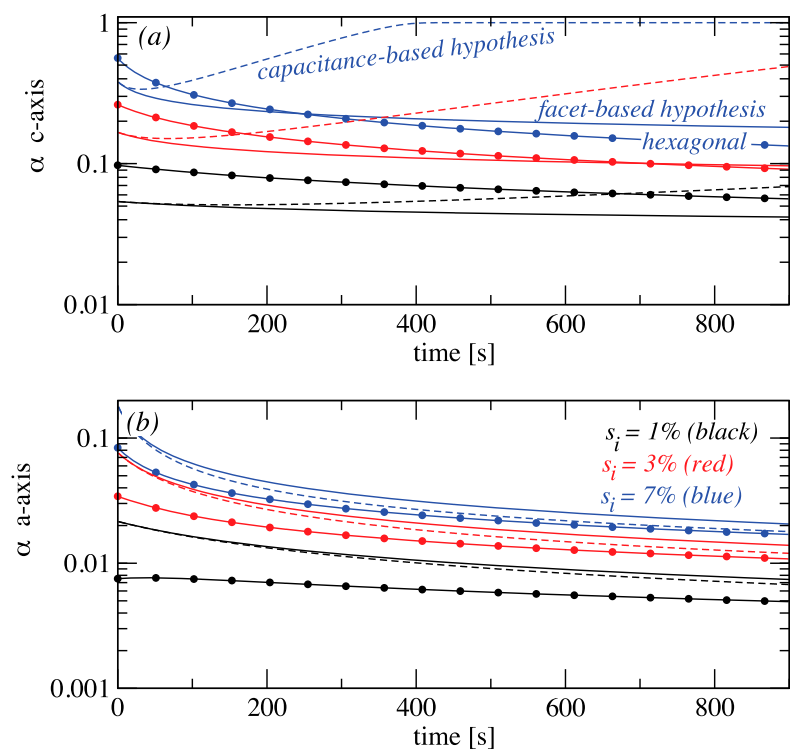

FIG. 8. Time series of (a) $c$-axis and (b) $a$-axis deposition coefficients for the simulations shown in Fig. 5 at $T=-7^{\circ} \mathrm{C}$. Lines with circles indicate hexagonal model solutions, and solid and dashed lines indicate DiSKICE solutions with the facet-based and aspectratio-based hypotheses, respectively.

growth as compared to the hexagonal growth model of Wood et al. (2001), no comparisons to growth data have been done. Few comprehensive datasets exist to which analytical growth models can be compared, and this is especially true at low ice supersaturations. However, a few datasets exist from wind tunnel measurements of crystals grown at liquid saturation. The comprehensive dataset of Fukuta and Takahashi (1999) is particularly useful because freely suspended crystals were grown for long periods of time (up to $30 \mathrm{~min}$ ) and data are reported for the axis lengths, crystal mass, and fall speed (reproduced in Figs. 10 and 11). Crystals grown in these experiments had a range of initial sizes, but the model simulations below use spheres with an initial radius of $10 \mu \mathrm{m}$ based on the studies of Sulia and Harrington (2011). All of the simulations below are integrated for up to $15 \mathrm{~min}$ at liquid saturation, and a constant temperature and pressure $(1000 \mathrm{hPa})$. Effective density, fall speed, and ventilation effects are computed following the axis-dependent approach described in Chen and Lamb (1994). Ventilation effects are particularly important here, as they strongly impact the growth rates for larger crystals. As discussed by Chen and Lamb (1994), ventilation effects tend to not only increase the overall mass growth rate, but the major axis growth rate is also amplified leading to thinner crystals.

Prior comparisons with the above data using the Chen and Lamb (1994) model showed that the mass, axis 


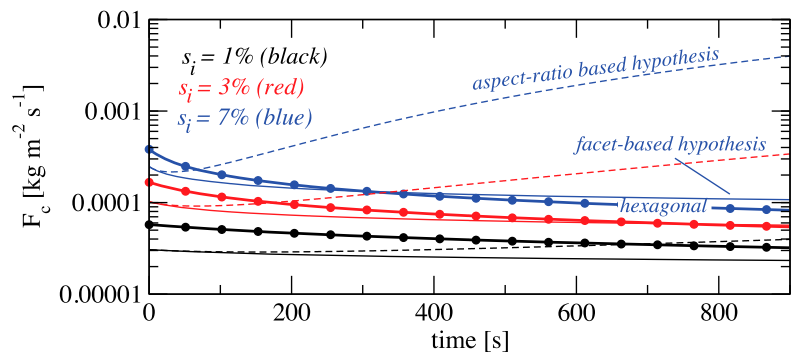

FIG. 9. Time series of the vapor flux onto the $c$ axis for the simulations shown in Fig. 5 at $T=-7^{\circ} \mathrm{C}$. Lines with circles indicate hexagonal model solutions, and solid and dashed lines indicate DiSKICE solutions with the facet-based and aspect-ratio-based hypotheses, respectively.

lengths, and fall speed could be captured with relatively high accuracy (Sulia and Harrington 2011; Harrington et al. 2013b). Typical results from those comparisons are reproduced in Figs. 10 and 11. These results provide a benchmark for the DiSKICE model at liquid saturation, and they also underscore an important point: Diffusionlimited growth captures the mass evolution of the observed crystals, whereas the deposition coefficient ratio $\Gamma$ primarily controls aspect-ratio evolution.

Simulations of crystal growth with DiSKICE used the facet-based hypothesis and ledge nucleation growth with $s_{\text {char }}$ from the polynomial fits given in Table 1 . The DiSKICE simulations produce results that are similar to those of the Chen and Lamb (1994) model and also compare well with the observed evolution of axis length (Fig. 10), mass, and fall speed (Fig. 11). Like the Chen and Lamb (1994) model, relative errors in the simulated crystal properties can often be large $(20 \%-50 \%$ for individual data points), but the general qualitative features of habit evolution and crystal fall speed are reproduced by DiSKICE: The model captures the strong increase in the $a$-axis length and the small $c$-axis length where thin dendritic crystals are observed near $-15^{\circ} \mathrm{C}$. These crystals have a low effective density (around $100 \mathrm{~kg} \mathrm{~m}^{-3}$ ) and a large area leading to low fall speeds. The model predicts the greatest fall speeds, and lowest masses, near the habit transition temperatures of $-10^{\circ}$ and $-22^{\circ} \mathrm{C}$, where crystals are relatively isometric with low drag and high effective density. Similar to the Chen and Lamb (1994) model, columns are predicted to be too thin with $a$-axis lengths that are smaller than observed.

Simulations assuming dislocation growth on the basal and prism facets produce crystals that are too thick in comparison to the measurements (Fig. 10). This result occurs because dislocations, unlike ledge nucleation, produce relatively high $\alpha$ along both axes (Fig. 12a). Consequently, both the $a$ and the $c$ axes grow with high efficiency.

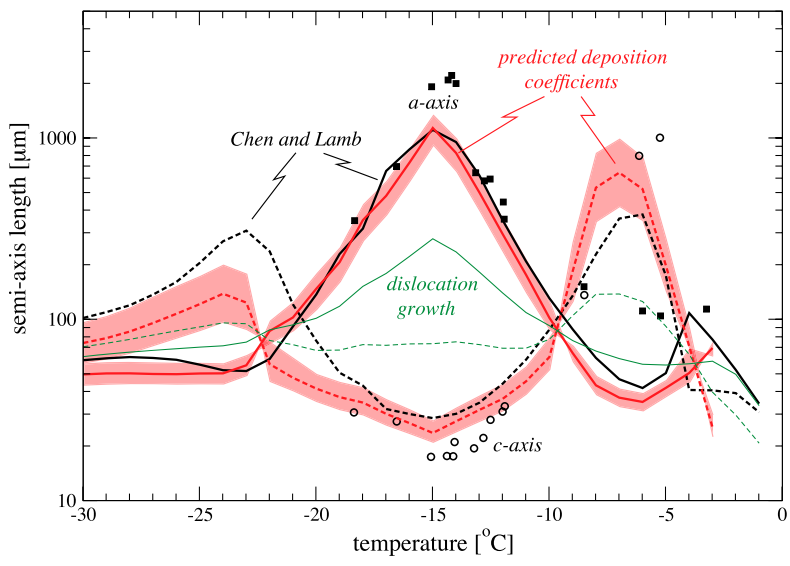

FIG. 10. Axis length after $15 \mathrm{~min}$ of growth at liquid saturation and 1000-hPa pressure as derived from wind tunnel data of Fukuta and Takahashi (1999) ( $a$ axis: solid circles; $c$ axis: open circles) and from model simulations ( $a$ axis: solid lines; $c$ axis: dashed lines). Simulations using the parameterization of Chen and Lamb (1994) are given by the black lines whereas simulations using predicted deposition coefficients (ledge nucleation, facet-based hypothesis) are given by the red lines. The red-shaded region indicates the range of uncertainty in the characteristic supersaturation $s_{\text {char }}$. Simulations with dislocation growth are indicated by the green lines.

There are two other possible mechanisms that could produce thin crystals. It is certainly possible that dislocation growth could occur on the primary growing axis, whereas ledge nucleation could occur on the weakly growing facet. However, simulations of this process produce crystals that are far too thin in comparison to the measurements (not shown). It is also possible that both dislocations and ledge nucleation occur on each facet, and that the growth mechanism with the largest $\alpha$ controls the growth (Nelson and Knight 1998). DiSKICE simulations with $\alpha$ chosen based on the most efficient growth mechanism produce thicker crystals reminiscent of dislocation growth. A key result of these simulations is that only ledge nucleation for each axis can reproduce crystal growth at liquid saturation. However, it should be borne in mind that real crystals may indeed grow by the aforementioned mechanisms, and that the inability of DiSKICE to reproduce those growth mechanisms may indicate a limitation of the model. Nevertheless, from a practical parameterization perspective, ledge nucleation can be used to reproduce the growth of thin crystals at liquid saturation.

It is curious that the model of Chen and Lamb (1994) and DiSKICE produce results that are similar to one another at liquid saturation even though the models are driven by different datasets. The ratio $\Gamma$ used in Chen and Lamb (1994) is derived from the $\alpha$ measurements of Lamb and Scott (1974), whereas DiSKICE uses polynomial fits to $s_{\text {char }}$ that are primarily due to the 

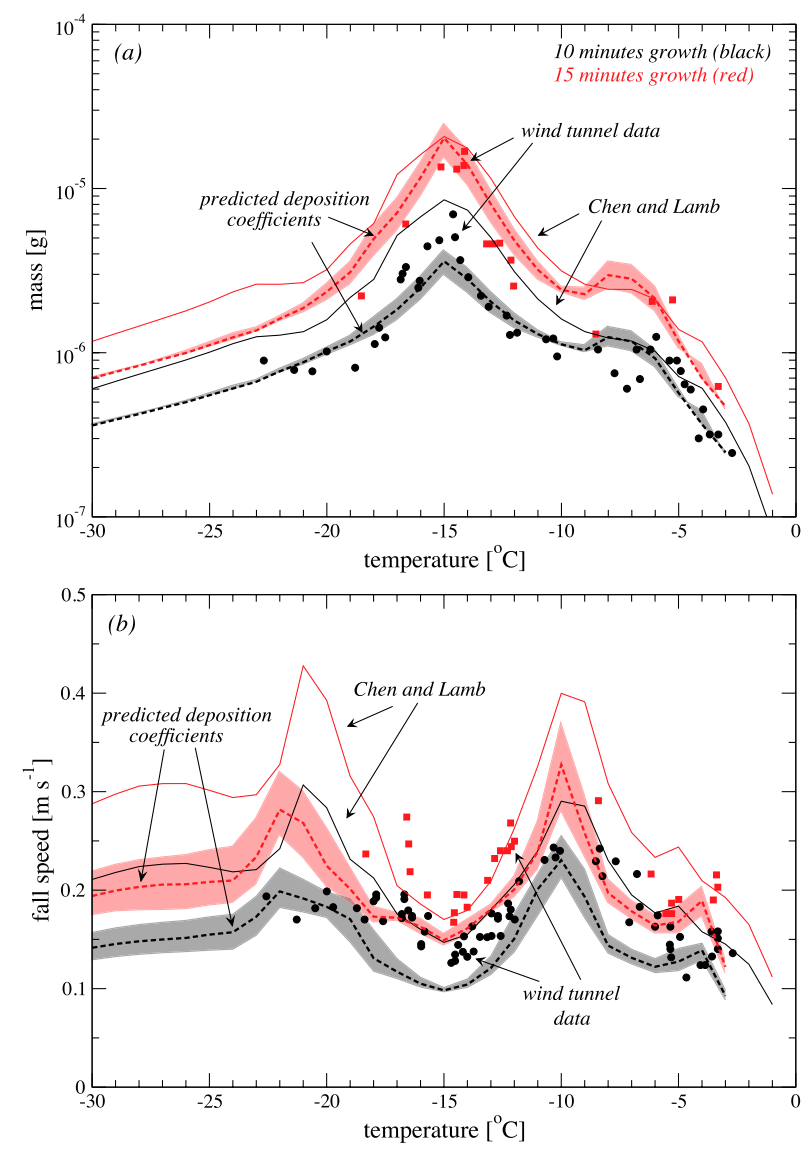

FIG. 11. (a) Ice mass and (b) fall speed after 10 (black) and 15 (red) min of growth at liquid saturation and 1000-hPa pressure. Wind tunnel data (Fukuta and Takahashi 1999) are indicated by the symbols and model simulations by the lines. Simulations using the Chen and Lamb (1994) parameterization are given by the solid lines whereas simulations using predicted deposition coefficients (ledge nucleation, facet-based hypothesis) are shown by the dashed lines. The shaded regions indicate the range of uncertainty in the characteristic supersaturation $s_{\text {char }}$.

measurements of Nelson and Knight (1998) at these temperatures. Since the models use different growth hypotheses, namely, the facet-based hypothesis for DiSKICE and the aspect-ratio-based hypothesis for Chen and Lamb (1994), the results presented above suggest that $\alpha_{c} / \alpha_{a}$ for ledge nucleation growth should approach $\Gamma(T) c / a$ in the limit of liquid saturation. While we have not discovered an analytical proof of this assertion, it is consistent with the model results. For instance, Fig. 12b shows the ratio $\alpha_{c} / \alpha_{a}$ and $\Gamma(T) c / a$ from the DiSKICE and Chen and Lamb (1994) simulations, respectively. The ratios are nearly identical after $15 \mathrm{~min}$ of growth. The near equality of these ratios provides circumstantial evidence for why the Chen and Lamb (1994) model is accurate at liquid saturation: The ratio $\Gamma(T) c / a$ provides a parameterization of the change in the
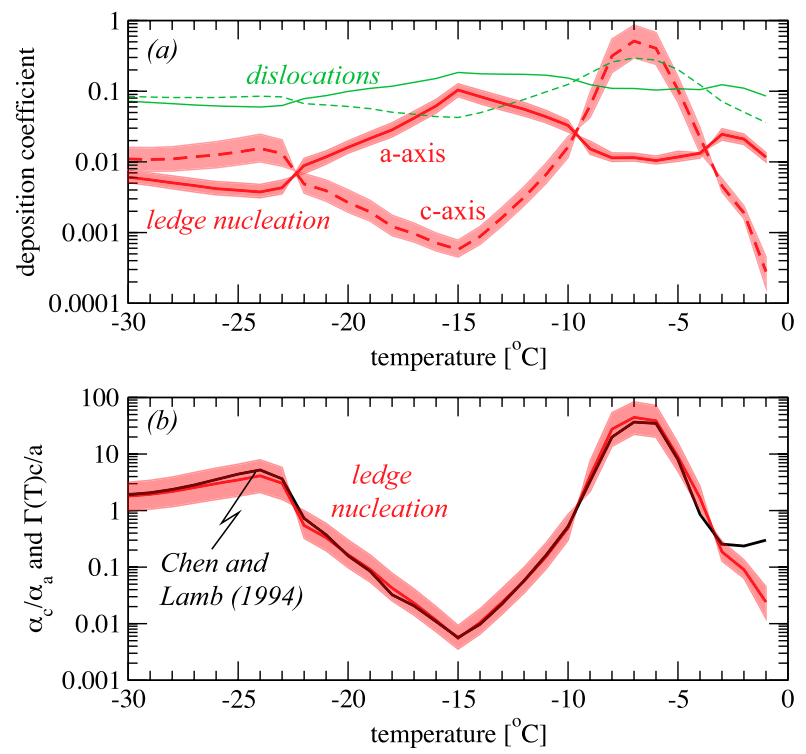

FIG. 12. (a) Deposition coefficients ( $a$ axis: solid lines; $c$ axis: dashed lines) after $15 \mathrm{~min}$ of growth at liquid saturation and 1000-hPa pressure for the simulations shown in Fig. 10. Simulations using ledge nucleation are given by the red lines and the red shaded region indicates the range of uncertainty in the characteristic supersaturation $s_{\text {char }}$. Simulations with dislocation growth are indicated by the green lines. (b) Ratio of the deposition coefficients $\left(\alpha_{c} / \alpha_{a}\right)$ for ledge nucleation growth (red line and shaded region) using the results and uncertainty from (a). The combination of the inherent growth ratio and the aspect ratio $[\Gamma(T) c / a]$ from the theory of Chen and Lamb (1994) is given by the black line.

deposition coefficient ratio. Moreover, these results provide a tantalizing hint of an underlying commonality in the measurements of Lamb and Scott (1974) and Nelson and Knight (1998).

\section{c. Aspect-ratio dependence on pressure}

In a series of experiments using a free-fall chamber Gonda (1976) measured the dependence of aspect ratio on the vapor diffusion coefficient $D_{v}$ at liquid saturation and temperatures of $-7^{\circ}$ and $-15^{\circ} \mathrm{C}$. Crystals formed from frozen liquid droplets fell about 5 to perhaps $15 \mathrm{~cm}$ onto a window where the crystals could be imaged. Measurements showed that the aspect ratios of the hexagonal crystals deviated further from unity for higher pressure or lower values of $D_{v}$ (Fig. 13), a result that is consistent with theory: The rate of vapor diffusion to a growing crystal depends on the background gas pressure. At relatively high pressure (low $D_{v}$ ), the diffusive resistance to vapor transport is large, keeping $s_{\text {surf }}$ below the value needed for minor axis growth. The aspect ratio of the crystal therefore evolves away from unity in time. Conversely, at very low pressures (high $D_{v}$ ) the background gas provides little resistance to the flow of vapor. In this case $s_{\text {surf }}$ is closer to the ambient 


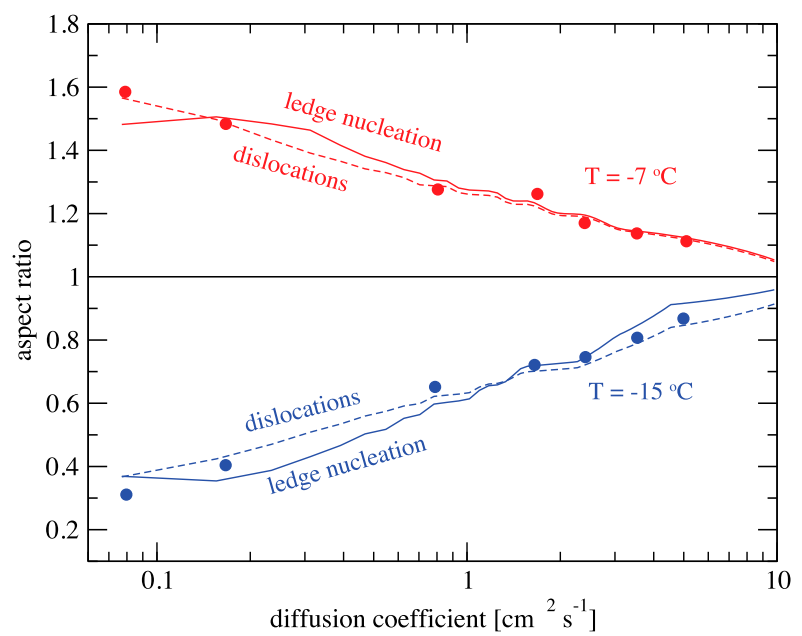

FIG. 13. Aspect ratio of crystals as a function of the vapor diffusivity $D_{v}$ at $-7^{\circ}$ and $-15^{\circ} \mathrm{C}$ (red and blue, respectively). Solid circles indicate the free-fall chamber measurements of Gonda (1976) made after crystal vapor growth at liquid saturation. Simulated crystals began as spheres with an initial radius of $2 \mu \mathrm{m}$ and grew during free-fall over a distance of $10 \mathrm{~cm}$. Dislocation growth (dashed line) used $a$ - and $c$-axis $s_{\text {char }}$ values, respectively, of $0.48 \%$ and $0.18 \%$ at $-7^{\circ} \mathrm{C}$ and $0.04 \%$ and $1.9 \%$ at $-15^{\circ} \mathrm{C}$. Ledge nucleation growth (solid line) used $a$ - and $c$-axis $s_{\text {char }}$ values, respectively, of $0.48 \%$ and $0.35 \%$ at $-7^{\circ} \mathrm{C}$ and $0.59 \%$ and $1.5 \%$ at $-15^{\circ} \mathrm{C}$.

value (liquid saturation), which is above $s_{\text {char }}$ for each axis, and therefore, isometric crystals are produced.

We simulated a scenario similar to the experiments of Gonda (1976) by allowing initially spherical crystals (radius of $2 \mu \mathrm{m}$, following Nelson 2001) to grow while falling $10 \mathrm{~cm}$. The model of Chen and Lamb (1994) cannot reproduce the dependence of crystal aspect ratio on $D_{v}$ since $\Gamma$ is constant (not shown). In contrast, the DiSKICE model produces an aspect-ratio dependence on $D_{v}$ that is similar to the measurements (Fig. 13). Moreover, either dislocation growth or ledge nucleation can fit the data though different $s_{\text {char }}$ values are required for each growth mechanism. At $-7^{\circ} \mathrm{C}$ dislocation growth requires $s_{\text {char }}$ for the basal and prism facets of $0.18 \%$ and $0.48 \%$, respectively, values that are similar to those from prior measurements $(0.2 \%$ and $0.44 \%$; Fig. 1). Ledge nucleation requires nearly the same $s_{\text {char }}$ for each axis, which is inconsistent with prior measurements. This result is consistent with Nelson (2001), who concluded that dislocation growth likely controlled the growth at $-7^{\circ} \mathrm{C}$. At $-15^{\circ} \mathrm{C}$, however, the $s_{\text {char }}$ required for ledge nucleation to fit the growth data $(0.59 \%$ and $1.5 \%$ for the prism and basal facets, respectively) are consistent with prior measurements $(0.54 \%$ and $2.1 \%$; Fig. 1). Dislocation growth requires $s_{\text {char }}$ for the basal facet to be an order of magnitude smaller than observed. This result is consistent with the findings of Nelson (2001) at low $D_{v}$, which indicated that ledge nucleation was occurring, but not at high $D_{v}$, where it appeared that dislocation growth occurred. The inconsistency may indicate a limitation of our model at higher $D_{v}$, but further laboratory studies are needed.

Because the experiments of Gonda (1976) produced only small crystals (less than $20 \mu \mathrm{m}$ ) formed from frozen droplets, it is likely that dislocation growth dominated much of the early growth of these crystals. However, explaining the thin crystals from the wind tunnel data of Fukuta and Takahashi (1999) requires ledge nucleation. Taken together, these results suggest that the early growth of small crystals may be dominated by dislocation growth while ledge nucleation dominates the growth at latter stages when crystals are large. This conclusion is broadly consistent with the discussions of Nelson (2001) and with the results of Gonda and Yamazaki (1984), who showed that crystals formed from frozen drops initially grow efficiently until facets become large enough that ledge nucleation dominates the growth.

\section{Summary and concluding remarks}

In this paper we have provided a composite dataset for the characteristic supersaturations $s_{\text {char }}$ that are needed as input for supersaturation-dependent $\alpha$. We have shown these $s_{\text {char }}$ values, when used in conjunction with the model of Zhang and Harrington (2014), can reproduce the growth of ice crystals at liquid saturation as observed in a wind tunnel. Only ledge nucleation for both axes is capable of reproducing the mass, lengths, and the fall speed of the measured crystals. Axis evolution based on the aspect-ratio-based hypothesis of Chen and Lamb (1994) produces columnar growth with unrealistic aspect ratios when $\alpha$ is predicted. This occurs because of a positive feedback with the aspect ratio in that parameterization method. Only the facet-based hypothesis of Nelson and Baker (1996) is capable of reproducing aspect-ratio evolution from a hexagonal model at both low and high supersaturations. While the diffusion-limited model of Chen and Lamb (1994) will not produce variations in aspect ratio with pressure, the model of Zhang and Harrington (2014) is capable of reproducing pressure-dependent growth of small crystals in comparison to measurements.

The growth of ice at low temperatures $\left(T<-30^{\circ} \mathrm{C}\right)$ has been infrequently measured, though the experiments of Libbrecht (2003b) indicate that the growth of basal and prism facets is driven primarily by ledge nucleation. However, no measurements of $s_{\text {char }}$ exist at temperatures below $-40^{\circ} \mathrm{C}$. To help fill this gap, we reanalyzed prior measurements (Magee et al. 2006) to extract average values of $s_{\text {char }}$ between $-40^{\circ}$ and $-60^{\circ} \mathrm{C}$. 
Comparisons to the normalized capacitance values of Bailey and Hallett (2010) for hexagonal plates and columns indicate that our values of $s_{\text {char }}$ are generally consistent with their data.

It is critical to bear in mind the approximate nature of the analyses at $T<-40^{\circ} \mathrm{C}$. At present, only two datasets have been published with precise measurements of facet growth down to $-40^{\circ} \mathrm{C}$, and both datasets indicate that $s_{\text {char }}$ increases with decreasing temperature (Libbrecht 2003b; Libbrecht and Rickerby 2013). While the $s_{\text {char }}$ values from our analysis are consistent with these measurements, it is not known whether our values are truly representative of the average growth of basal and prism facets. The morphology of crystals grown in the studies of Magee et al. (2006) is unknown, and since those crystals were formed from frozen droplets they likely were polycrystalline (Bacon et al. 2003), may have had mesoscopic surface features (Magee et al. 2014), and likely underwent a transition as facets emerged. These processes, and others, would be convolved together in the values of $s_{\text {char }}$ that we have derived.

It is also difficult to relate these known growth mechanisms, and measured growth rates, to the measures of crystal roughness reported in the literature (Neshyba et al. 2013; Magee et al. 2014; Schnaiter et al. 2016). Magee et al. (2014) showed mesoscopic features on crystal facets, yet growth was at times limited by attachment kinetics. Moreover, Pedersen et al. (2011) found weak growth of crystal facets until a grain boundary is formed through the contact of two dissimilar facets. More recently, Voigtländer et al. (2018) indicated that crystals cycled between growth and sublimation show reduced growth rates in later cycles, and that surface roughening can increase during cycled growth. This latter result is consistent with prior measurements that show faceting disappears and crystals roughen during sublimation (Nelson 1998; Magee et al. 2014). Taken together, these results indicate that our understanding of ice vapor growth is still in its infancy. Approximate models, such as the one posed in this paper, must be used with caution and should be interpreted as a placeholder for a more precise theory of ice growth.

Acknowledgments. The authors are grateful for support from the National Science Foundation through Grants AGS-1433201 and AGS-1824243. Stimulating conversations with Dr. Dennis Lamb were, as always, insightful and useful. The first author also benefited from discussions with Drs. Jon Nelson and Brian Swanson. This article benefited greatly from the careful review of its contents by two anonymous reviewers and a review by Dr. Andrew Heymsfield; for this the authors are thankful.

\section{REFERENCES}

Asakawa, H., G. Sazaki, E. Yokoyama, K. Ngashima, and S. Nakatsubo, 2014: Roles of surface/volume diffusion in the growth kinetics of elementary spiral steps on ice basal faces grown from water vapor. Cryst. Growth Des., 14, 3210-3220, https://doi.org/10.1021/cg4014653.

Avramov, A., and J. Y. Harrington, 2010: The influence of parameterized ice habit on simulated mixed-phase Arctic clouds. J. Geophys. Res., 115, D03205, https://doi.org/10.1029/2009JD012108.

Bacon, N., M. Baker, and B. Swanson, 2003: Initial stages in the morphological evolution of vapour-grown ice crystals: A laboratory investigation. Quart. J. Roy. Meteor. Soc., 129, 1903-1927, https://doi.org/10.1256/qj.02.04.

Bailey, M., and J. Hallett, 2004: Growth rates and habits of ice crystals between $-20^{\circ}$ and $-70^{\circ}$ C. J. Atmos. Sci., 61, 514-544, https://doi.org/10.1175/1520-0469(2004)061<0514:GRAHOI> 2.0. $\mathrm{CO} ; 2$

— and - 2010: Laboratory measured ice crystal capacitances and mass dimensional relations. 13th Conf. on Cloud Physics, Portland, OR, Amer. Meteor. Soc., P1.30, https://ams.confex.com/ ams/pdfpapers/171204.pdf.

Bartels-Rausch, T., and Coauthors, 2014: A review of air-ice chemical and physical interactions (AICI): Liquids, quasiliquids, and solids in snow. Atmos. Chem. Phys., 14, 15871633, https://doi.org/10.5194/acp-14-1587-2014.

Burton, W. K., N. Cabrera, and F. C. Frank, 1951: The growth of crystals and the equilibrium structure of their surfaces. Philos. Trans. Roy. Soc. London, 243A, 299-358, https://doi.org/ 10.1098/rsta.1951.0006.

Carignano, M., 2007: Formation of stacking faults during ice growth on hexagonal and cubic substrates. J. Phys. Chem. Lett., 111, 501-504, https://doi.org/10.1021/jp067388q.

Chen, J.-P., and D. Lamb, 1994: The theoretical basis for the parameterization of ice crystal habits: Growth by vapor deposition. J. Atmos. Sci., 51, 1206-1221, https://doi.org/10.1175/ 1520-0469(1994)051<1206:TTBFTP>2.0.CO;2.

- and - 1999: Simulation of cloud microphysical and chemical processes using a multicomponent framework. Part II: Microphysical evolution of a wintertime orographic cloud. J. Atmos. Sci., 56, 2293-2312, https://doi.org/10.1175/15200469(1999)056<2293:SOCMAC $>2$ 2.0.CO;2.

_, and T.-C. Tsai, 2016: Triple-moment modal parameterization for the adaptive growth habit of pristine ice crystals. J. Atmos. Sci., 73, 2105-2122, https://doi.org/10.1175/JAS-D-15-0220.1.

Choularton, T., and J. Latham, 1977: Measurements of the deposition coefficient for ice, and its application to cirrus seeding. Quart. J. Roy. Meteor. Soc., 103, 307-318, https://doi.org/ 10.1002/qj.49710343608.

Connolly, P., C. Emersic, and P. Field, 2012: A laboratory investigation into the aggregation efficiency of small ice crystals. Atmos. Chem. Phys., 12, 2055-2076, https://doi.org/10.5194/ acp-12-2055-2012.

Constantin, J. G., M. M. Gianetti, M. P. Longinotti, and H. R. Corti, 2018: The quasi-liquid layer of ice revisited: The role of temperature gradients and tip chemistry in AFM studies. Atmos. Chem. Phys., 18, $14965-14$ 978, https://doi.org/10.5194/ acp-18-14965-2018.

Elbaum, M., 1991: Roughening transition observed on the prism facet of ice. Phys. Rev. Lett., 67, 2982-2985, https://doi.org/ 10.1103/PhysRevLett.67.2982.

Frank, F., 1982: Snow crystals. Contemp. Phys., 23, 3-22, https:// doi.org/10.1080/00107518208231565. 
Fukuta, N., 1969: Experimental studies on the growth of small ice crystals. J. Atmos. Sci., 26, 522-531, https://doi.org/10.1175/ 1520-0469(1969)026<0522:ESOTGO>2.0.CO;2.

—, and T. Takahashi, 1999: The growth of atmospheric ice crystals: A summary of findings in vertical supercooled cloud tunnel studies. J. Atmos. Sci., 56, 1963-1979, https://doi.org/ 10.1175/1520-0469(1999)056<1963:TGOAIC $>2.0$. CO;2.

Furukawa, Y., and T. Kobayashi, 1978: On the growth mechanism of polycrystalline snow crystals with a specific grain boundary. J. Cryst. Growth, 45, 57-65, https://doi.org/10.1016/00220248(78)90415-3.

Gierens, K., M. Monier, and J.-F. Gayet, 2003: The deposition coefficient and its role for cirrus. J. Geophys. Res., 108, 4069, https://doi.org/10.1029/2001JD001558.

Gonda, T., 1976: The growth of small ice crystals in gases of low and high pressures. J. Meteor. Soc. Japan, 54, 233-240, https:// doi.org/10.2151/jmsj1965.54.4_233.

, and T. Yamazaki, 1978: Morphology of ice droxtals grown from supercooled water droplets. J. Cryst. Growth, 45, 66-69, https://doi.org/10.1016/0022-0248(78)90416-5.

$\ldots$, and — 1984: Initial growth forms of snow crystals growing from frozen cloud droplets. J. Meteor. Soc. Japan, 62, 190-192, https://doi.org/10.2151/jmsj1965.62.1_190.

_ - Y. Matsuura, and T. Sie, 1994: In situ observation of vapor-grown ice crystals by laser two-beam interferometry. J. Cryst. Growth, 142, 171-176, https://doi.org/10.1016/0022-0248(94)90285-2.

Ham, F., 1959: Shape-preserving solutions of the time-dependent diffusion equation. Quart. Appl. Math., 17, 137-145, https:// doi.org/10.1090/qam/108196.

Harrington, J., K. Sulia, and H. Morrison, 2013a: A method for adaptive habit prediction in bulk microphysical models. Part I: Theoretical development. J. Atmos. Sci., 70, 349-364, https:// doi.org/10.1175/JAS-D-12-040.1.

_ — - and — 2013b: A method for adaptive habit prediction in bulk microphysical models. Part II: Parcel model corroboration. J. Atmos. Sci., 70, 365-376, https://doi.org/ 10.1175/JAS-D-12-0152.1.

Harrison, A., A. Moyle, M. Hanson, and J. Harrington, 2016: Levitation diffusion chamber measurements of the mass growth of small ice crystals from vapor. J. Atmos. Sci., 73, 2743-2758, https://doi.org/10.1175/JAS-D-15-0234.1.

Hashino, T., and G. J. Tripoli, 2007: The Spectral Ice Habit Prediction System (SHIPS). Part I: Model description and simulation of the vapor deposition process. J. Atmos. Sci., 64, 2210-2237, https://doi.org/10.1175/JAS3963.1.

Jensen, A., J. Harrington, H. Morrison, and J. Milbrandt, 2017: Predicting ice shape evolution in a bulk microphysics model. $J$. Atmos. Sci., 74, 2081-2104, https://doi.org/10.1175/JAS-D-16-0350.1.

Kobayashi, T., Y. Furukawa, K. Kikuchi, and H. Uyeda, 1976: On twinned structures in snow crystals. J. Cryst. Growth, 32, 233 249, https://doi.org/10.1016/0022-0248(76)90038-5.

Kong, X., E. Thomson, P. Papagiannakopoulos, S. Johansson, and J. Petterson, 2014: Water accommodation on ice and organic surfaces: Insights from environmental molecular beam experiments. J. Phys. Chem., 118, 13 378-13 386, https://doi.org/ 10.1021/jp5044046.

Kuhs, W., C. Sippel, A. Falenty, and T. Hansen, 2012: Extent and relevance of stacking disorder in "ice $\mathrm{I}_{\mathrm{c}}$." Proc. Natl. Acad. Sci. USA, 109, 21 259-21 264, https://doi.org/10.1073/ pnas. 1210331110.

Kuroda, T., and R. Lacmann, 1982: Growth kinetics of ice from the vapor phase and its growth forms. J. Cryst. Growth, 56, 189205, https://doi.org/10.1016/0022-0248(82)90028-8.
Lamb, D., 2000: Crystal growth: 2-D or not 2-D? 13th Int. Conf. on Clouds and Precipitation, Reno, NV, International Commission on Clouds and Precipitation, 9.36.

— , and W. Scott, 1974: The mechanism of ice crystal growth and habit formation. J. Atmos. Sci., 31, 570-580, https://doi.org/ 10.1175/1520-0469(1974)031<0570:TMOICG >2.0.CO;2.

— of ice crystals by vapor deposition. Preprints, Conf. on Cloud Physics, Dallas, TX, Amer. Meteor. Soc., 389-392.

Libbrecht, K., 2003a: Explaining the formation of thin ice crystal plates with structure-dependent attachment kinetics. J. Cryst. Growth, 258, 168-175, https://doi.org/10.1016/S0022-0248(03)01496-9.

- 2003b: Growth rates of the principal facets of ice between $-10^{\circ} \mathrm{C}$ and $-40^{\circ} \mathrm{C}$. J. Cryst. Growth, 247, 530-540, https://doi.org/10.1016/S0022-0248(02)01996-6.

_ kinetics for faceted ice crystal growth. J. Cryst. Growth, 377, 1-8, https://doi.org/10.1016/j.jcrysgro.2013.04.037.

MacKenzie, A., and P. Haynes, 1992: The influence of surface kinetics on the growth of stratospheric ice crystals. J. Geophys. Res., 97, 8057-8064, https://doi.org/10.1029/91JD01436.

Magee, N., 2006: A laboratory investigation of vapor-grown ice crystals at low atmospheric temperatures. Ph.D. thesis, The Pennsylvania State University, 234 pp.

— A. Moyle, and D. Lamb, 2006: Experimental determination of the deposition coefficient of small cirrus-like crystals near $-50^{\circ} \mathrm{C}$. Geophys. Res. Lett., 33, L17813, https:// doi.org/10.1029/2006GL026665.

— K. Spector, Y.-H. Lin, C. Tong, and J. Beatty, 2011: Initial ice microparticle sublimation measurements from the Levitating Upper-Tropospheric Environmental Simulator (LUTES). J. Atmos. Oceanic Technol., 28, 884-890, https://doi.org/ 10.1175/JTECH-D-11-00028.1.

— A. Miller, M. Amaral, and A. Cumiskey, 2014: Mesoscopic surface roughness of ice crystals pervasive across a wide range of ice crystal conditions. Atmos. Chem. Phys., 14, $12357-$ 12 371, https://doi.org/10.5194/acp-14-12357-2014.

Miller, T., and K. Young, 1979: A numerical simulation of ice crystal growth from the vapor phase. J. Atmos. Sci., 36, 458469, https://doi.org/10.1175/1520-0469(1979)036<0458:ANSOIC > 2.0.CO;2.

Nelson, J., 1993: Heat conduction problems in crystal growth from the vapor. J. Cryst. Growth, 132, 538-550, https://doi.org/ 10.1016/0022-0248(93)90082-8.

- 1994: A theoretical study of ice crystal growth in the atmosphere. Ph.D. thesis, University of Washington, 183 pp.

_ 1998: Sublimation of ice crystals. J. Atmos. Sci., 55, 910-919, https://doi.org/10.1175/1520-0469(1998)055<0910:SOIC > 2.0.CO;2.

- 2001: Growth mechanisms to explain the primary and secondary habits of snow crystals. Philos. Mag., 81A, 2337-2373, https://doi.org/10.1080/01418610108217152.

_ 2005: Interactive comment on "Supersaturation dehydration, and denitrification in Arctic cirrus" by B. Kärcher. Atmos. Chem. Phys. Discuss., 5, S257-S260, https://www.atmos-chemphys-discuss.net/5/S545/2005/acpd-5-S545-2005.pdf.

— of vapor growth and sublimation of small ice crystals in the atmosphere. J. Geophys. Res., 101, 7033-7047, https://doi.org/ 10.1029/95JD03162.

, and C. Knight, 1998: Snow crystal habit changes explained by layer nucleation. J. Atmos. Sci., 55, 1452-1465, https://doi.org/ 10.1175/1520-0469(1998)055<1452:SCHCEB >2.0.CO;2. 
_- and B. Swanson, 2018: How the protruding growth mechanism may produce corner pockets and other features on snow crystals. 15th Conf. on Cloud Physics, Vancouver, BC, Canada, Amer. Meteor. Soc., 64, https://ams.confex.com/ ams/15CLOUD15ATRAD/meetingapp.cgi/Paper/346283.

Neshyba, S., B. Lowen, M. Benning, A. Lawson, and P. Rowe, 2013: Roughness metrics of prismatic facets of ice. J. Geophys. Res. Atmos., 118, 3309-3318, https://doi.org/ 10.1002 /jgrd.50357.

Pedersen, C., A. Mihranyan, and M. Stromme, 2011: Surface transition on ice induced by the formation of a grain boundary. PLOS ONE, 6, e24373, https://doi.org/10.1371/ journal.pone.0024373.

Pfalzgraff, W., R. M. Hulscher, and S. P. Neshyba, 2010: Scanning electron microscopy and molecular dynamics of surfaces of growing and ablating hexagonal ice crystals. Atmos. Chem. Phys., 10, 2927-2935, https://doi.org/10.5194/acp-102927-2010.

Pokrifka, G., 2018: Using laboratory measurements of vaporgrown ice crystals to infer surface kinetics and estimate the deposition coefficient. M.S. thesis, Dept. of Meteorology and Atmospheric Science, The Pennsylvania State University, $89 \mathrm{pp}$.

Schnaiter, M., and Coauthors, 2016: Cloud chamber experiments on the origin of ice crystal complexity in cirrus clouds. Atmos. Chem. Phys., 16, 5091-5110, https://doi.org/ 10.5194/acp-16-5091-2016.

Sei, T., and T. Gonda, 1989: The growth mechanism and the habit change of ice crystals growing from the vapor phase. J. Cryst. Growth, 94, 697-707, https://doi.org/10.1016/0022-0248(89) 90094-8.

Skrotzki, J., and Coauthors, 2013: The accommodation coefficient of water molecules on ice-Cirrus cloud studies at the AIDA simulation chamber. Atmos. Chem. Phys., 13, 4451-4466, https://doi.org/10.5194/acp-13-4451-2013.

Sulia, K., and J. Harrington, 2011: Ice aspect ratio influences on mixed-phase clouds: Impacts on phase partitioning in parcel models. J. Geophys. Res., 116, D21309, https://doi.org/10.1029/ 2011JD016298.

Thompson, G., P. Field, R. Rasmussen, and W. Hall, 2008: Explicit forecasts of winter precipitation using an improved bulk microphysics scheme. Part II: Implementation of a new snow parameterization. Mon. Wea. Rev., 136, 5095-5115, https:// doi.org/10.1175/2008MWR2387.1.

Voigtländer, J., and Coauthors, 2018: Surface roughness during depositional growth and sublimation of ice crystals. Atmos. Chem. Phys., 18, 13 687-13 702, https://doi.org/10.5194/acp-18-13687-2018.

Westbrook, C. D., and A. J. Heymsfield, 2011: Ice crystals growing from vapor in supercooled clouds between $-2.5^{\circ}$ and $-20^{\circ} \mathrm{C}$ : Testing current parameterization methods using laboratory data. J. Atmos. Sci., 68, 2416-2430, https://doi.org/10.1175/ JAS-D-11-017.1.

_ R. J. Hogan, and A. J. Illingworth, 2008: The capacitance of pristine ice crystals and aggregate snowflakes. J. Atmos. Sci., 65, 206-219, https://doi.org/10.1175/2007JAS2315.1.

Wood, S., M. Baker, and D. Calhoun, 2001: New model for the vapor growth of hexagonal ice crystals in the atmosphere. J. Geophys. Res., 106, 4845-4870, https://doi.org/10.1029/ 2000JD900338.

Zhang, C., and J. Harrington, 2014: Including surface kinetic effects in simple models of ice vapor diffusion. J. Atmos. Sci., 71, 372-390, https://doi.org/10.1175/JAS-D-13-0103.1. , and - 2015: The effects of surface kinetics on crystal growth and homogeneous freezing in parcel simulations of cirrus. J. Atmos. Sci., 72, 2929-2946, https://doi.org/10.1175/ JAS-D-14-0285.1. 